Article

\title{
Effects of Rich in B-Glucans Edible Mushrooms on Aging Gut Microbiota Characteristics: An In Vitro Study
}

\author{
Evdokia K. Mitsou ${ }^{1}$, Georgia Saxami ${ }^{1}$, Emmanuela Stamoulou ${ }^{1}$, Evangelia Kerezoudi ${ }^{1}$, \\ Eirini Terzi ${ }^{1}$, Georgios Koutrotsios ${ }^{2}{ }^{\circledR}$, Georgios Bekiaris ${ }^{2}{ }^{\circledR}$, Georgios I. Zervakis ${ }^{2}{ }^{\circledR}$, \\ Konstantinos C. Mountzouris ${ }^{3}$, Vasiliki Pletsa ${ }^{4}$ and Adamantini Kyriacou ${ }^{1, *}$ \\ 1 Department of Nutrition and Dietetics, Harokopio University, 17671 Athens, Greece; \\ emitsou@hua.gr (E.K.M.); gsaxami@hua.gr (G.S.); dp4421749@hua.gr (E.S.); dp4421804@hua.gr (E.K.); \\ dp4521704@hua.gr (E.T.) \\ 2 Laboratory of General and Agricultural Microbiology, Department of Crop Science, \\ Agricultural University of Athens, 11855 Athens, Greece; georgioskoutrotsios@gmail.com (G.K.); \\ giorgosbekiaris@yahoo.gr (G.B.); zervakis@aua.gr (G.I.Z.) \\ 3 Department of Nutritional Physiology and Feeding, Agricultural University of Athens, \\ 11855 Athens, Greece; kmountzouris@aua.gr \\ 4 Institute of Chemical Biology, National Hellenic Research Foundation, 11635 Athens, Greece; vpletsa@eie.gr \\ * Correspondence: mkyriacou@hua.gr; Tel.: +30-210-9549142
}

Academic Editor: De-Xing Hou

Received: 26 May 2020; Accepted: 16 June 2020; Published: 18 June 2020

\begin{abstract}
Alterations of gut microbiota are evident during the aging process. Prebiotics may restore the gut microbial balance, with $\beta$-glucans emerging as prebiotic candidates. This study aimed to investigate the impact of edible mushrooms rich in $\beta$-glucans on the gut microbiota composition and metabolites by using in vitro static batch culture fermentations and fecal inocula from elderly donors $(\mathrm{n}=8)$. Pleurotus ostreatus, P. eryngii, Hericium erinaceus and Cyclocybe cylindracea mushrooms derived from various substrates were examined. Gut microbiota composition (quantitative PCR (qPCR)) and short-chain fatty acids (SCFAs; gas chromatography (GC)) were determined during the 24-h fermentation. P. eryngii induced a strong lactogenic effect, while P. ostreatus and C. cylindracea induced a significant bifidogenic effect $(p$ for all $<0.05$ ). Furthermore, P. eryngii produced on wheat straw and the prebiotic inulin had comparable Prebiotic Indexes, while P. eryngii produced on wheat straw/grape marc significantly increased the levels of tested butyrate producers. P. ostreatus, P. eryngii and C. cylindracea had similar trends in SCFA profile; H. erinaceus mushrooms were more diverse, especially in the production of propionate, butyrate and branched SCFAs. In conclusion, mushrooms rich in $\beta$-glucans may exert beneficial in vitro effects in gut microbiota and/or SCFAs production in elderly subjects.
\end{abstract}

Keywords: gut microbiota; SCFAs; aging; prebiotics; $\beta$-glucans; edible mushroom; Pleurotus ostreatus; Pleurotus eryngii; Hericium erinaceus; Cyclocybe cylindracea

\section{Introduction}

Gut microbiota, one of the most complex and dense microbial ecosystems, is recognized for its crucial role on human health through a variety of functions, such as the extraction of energy from foods, alterations in the appetite signaling pathway [1,2], involvement in host metabolic processes [3,4], host protection against pathogenic microorganisms [5,6] and immune system development and function [7]. Imbalance in the dynamic interactions among microbial intestinal populations, a phenomenon called dysbiosis, has been related to many pathological conditions, e.g., inflammatory bowel disease, irritable bowel 
syndrome, obesity, colorectal cancer [8,9]. The restoration of the normal intestinal microbiota can be acquired through the consumption of dietary factors, such as probiotics, prebiotics or synbiotics [10]. Prebiotics are defined as 'nondigestible food ingredients that when administered, exert a beneficial effect on the host health' [11]. They advance the growth of lactic acid bacteria and bifidobacteria in the colon, while exerting antagonistic action on harmful microorganisms [11]. The most recognized prebiotics are fructo-oligosaccharides (FOS), galacto-oligosaccharides (GOS), lactulose and inulin [12].

The beneficial effects of prebiotics have fueled the research for novel and alternative sources. Among the emerging prebiotic candidates are $\beta$-glucans, i.e., polysaccharides receiving increasing attention due to their human health benefits $[13,14]$. B-glucans are abundant in the bran of certain cereal grains (oat and barley), and also in various mushroom species $[15,16]$. Fungal $\beta$-glucans are beta-( $1 \rightarrow 3,1 \rightarrow 6)$-D-glucans, whereas their cereal-derived counterparts are beta-(1 $\rightarrow 3 / 1 \rightarrow 4)$-D-glucans [17].

Fermentation of prebiotics by intestinal microbial consortia results in the increased production of short-chain fatty acids (SCFAs), with key roles in the prebiotic function [18]. Acetate, propionate and butyrate are the most abundant $(\geq 95 \%)$ SCFAs in the human colon and stool. These microbial metabolites have been associated with many beneficial effects on host health, such as the inhibition of $\mathrm{pH}$-sensitive pathogens, the increase of mineral absorption, the regulation of intestinal motility and the enhancement of the intestinal epithelial barrier $[18,19]$. Acetate is transported from the gut by the portal vein in the liver, where it participates in lipogenesis, and also in distant body sites, and it can be metabolized in the human muscle, kidney, heart and brain [20]. Propionate exerts anti-lipogenic, cholesterol-lowering, anti-inflammatory and anti-carcinogenic activities [21]. Butyrate is the main energy source for intestinal epithelial cells, while it also modulates the cell growth and differentiation of intestinal cells, exerts strong anti-inflammatory activity, stimulates the cell apoptosis and exhibits strong anticancer properties [22-24].

In vitro intestinal fermentation models have been developed as a powerful tool for examining the impact of prebiotic substrates on the composition and metabolic activity of gut microbial populations [25]. There are several studies investigating the prebiotic potential using fecal inoculum from infants or healthy adults, but there are only a few relevant data for elderly populations [26]. In addition, only a few in vitro fermentation models have been developed in order to evaluate the effects of edible mushrooms (i.e., lyophilized powder from the entire fruitbody) [27], whereas several exist about the study of mushrooms extracts [28-30], or mushrooms $\beta$-glucans [31,32], on the composition and/or activity of the gut microbiota.

The aim of this work was to investigate the impact of rich in $\beta$-glucans edible mushrooms (derived from six strains of Cyclocybe cylindracea, Hericium erinaceus, Pleurotus eryngii and P. ostreatus isolated from Greek habitats and cultivated in various substrates) on the gut microbiota composition and metabolites production using an in vitro batch culture fermentation system inoculated with fecal samples from apparently healthy volunteers over 65 years old.

\section{Results}

Fungal strains and mushroom cultivation substrates appear in Table 1, together with the mushrooms content in total, $\alpha$ - and $\beta$-glucans. On the basis of the results presented, the mushrooms were rich in $\beta$-glucans, with a mean content of $31.58 \%(w / w$ d.w.) and a range from $15.4 \%$ in the case of HEBS up to $42.2 \%$ ( $w / w$ d.w.) in the case of PEWSGM. For the in vitro static batch culture fermentation, lyophilized mushroom powder was added to the basal medium, while the prebiotic inulin (INU2) was used as positive control, (a negative control (NC) was also included; i.e., a basal medium with no carbohydrate source). This procedure was repeated eight times, using fresh fecal inoculum from apparently healthy volunteers over 65 years old. Descriptive characteristics of the fecal donors are available in Table 2. All donors were compliant with the inclusion criteria of the study, concerning health status, dietary habits or the consumption of probiotics/prebiotics/antibiotics. Drug-treated hypertension was the most frequent medical condition among donors. No allergies or recent consumption of nonsteroidal anti-inflammatory drugs was reported among volunteers. 
Furthermore, most subjects were normal-weighted (62.5\%), moderate active $(75.0 \%)$, with low daily fiber intake $\left(75 \%\right.$ of cases below recommended intake of $\left.25-30 \mathrm{~g} \cdot \mathrm{d}^{-1}\right)$.

Faecal inocula were categorized as Bristol Stool Scale (BBS) type 5 in 4 cases, with the rest being assigned in type $3(n=1)$ and $4(n=3)$; no volunteer reported diarrheic evacuations for a $7-d$ period before sampling day, whereas chronic constipation or gastrointestinal problems were part of exclusion criteria.

Table 1. Fungal strains, mushroom cultivation substrates and glucans content *

\begin{tabular}{|c|c|c|c|c|}
\hline Description & Abbreviation & $\begin{array}{l}\text { Total Glucans } \\
\quad(\% w / w)\end{array}$ & $\begin{array}{l}\alpha \text {-Glucans } \\
(\% w / w)\end{array}$ & $\begin{array}{l}\beta \text {-Glucans } \\
(\% w / w)\end{array}$ \\
\hline $\begin{array}{l}\text { Pleurotus ostreatus IK } 1123 \text { in } 100 \% \text { wheat straw } \\
\text { (WS, control substrate) }\end{array}$ & POWS & $39.4 \pm 1.4$ & $8.7 \pm 1.3$ & $30.6 \pm 1.9$ \\
\hline Pleurotus ostreatus IK 1123 in olive pruning residues (OL) & POOLRP & $38.5 \pm 2.1$ & $3.4 \pm 0.2$ & $35.1 \pm 1.1$ \\
\hline $\begin{array}{l}\text { Pleurotus ostreatus LGM } 22 \text { in } 100 \% \text { wheat straw (control } \\
\text { substrate) }\end{array}$ & POLWS & $34.3 \pm 2.7$ & $6.6 \pm 1.1$ & $27.7 \pm 2.4$ \\
\hline $\begin{array}{l}\text { Pleurotus ostreatus LGM } 22 \text { in OL:TPOMW (ratio 3:1, w/w) } \\
\text { (TPOMW, two-phase olive mill wastes) }\end{array}$ & POLTPOMW & $39.9 \pm 0.8$ & $4.9 \pm 1.0$ & $35.0 \pm 0.4$ \\
\hline $\begin{array}{l}\text { Pleurotus eryngii LGAM } 216 \text { in 100\% wheat straw } \\
\text { (control substrate) }\end{array}$ & PEWS & $46.6 \pm 3.9$ & $7.9 \pm 1.3$ & $38.7 \pm 5.4$ \\
\hline $\begin{array}{l}\text { Pleurotus eryngii LGAM } 216 \text { in WS:GM } \\
\quad \text { (ratio } 1: 1, w / w) \text { (GM, grape marc) }\end{array}$ & PEWSGM & $49.7 \pm 2.9$ & $7.6 \pm 1.4$ & $42.2 \pm 5.9$ \\
\hline $\begin{array}{l}\text { Hericium erinaceus LGAM } 4514 \text { in } 100 \% \text { beech sawdust } \\
\text { (BS, control substrate) }\end{array}$ & HEBS & $16.4 \pm 0.1$ & $1.0 \pm 0.1$ & $15.4 \pm 0.2$ \\
\hline Hericium erinaceus LGAM 4514 in olive pruning residues & HEOLRP & $21.8 \pm 0.9$ & $1.1 \pm 0.1$ & $20.7 \pm 0.3$ \\
\hline $\begin{array}{l}\text { Cyclocybe cylindracea LGAM } 951 \text { in } 100 \% \text { wheat straw } \\
\text { (control substrate) }\end{array}$ & CC2WS & $39.3 \pm 1.7$ & $6.1 \pm 0.5$ & $33.2 \pm 1.9$ \\
\hline $\begin{array}{l}\text { Cyclocybe cylindracea LGAM } 961 \text { in } 100 \% \text { wheat straw } \\
\text { (control substrate) }\end{array}$ & CC505WS & $40.6 \pm 2.9$ & $3.4 \pm 1.1$ & $37.2 \pm 3.8$ \\
\hline
\end{tabular}

* Values are expressed as mean and standard deviation (SD) of \% w/w of dry weight $($ d.w. $)(n=4)$.

Table 2. Descriptive characteristics of fecal donors $(n=8)$.

\begin{tabular}{|c|c|}
\hline Sociodemographic Parameters & \\
\hline Sex (no. of males/females), n (\%) & $4 / 4(50.0 \% / 50.0 \%)$ \\
\hline Age (years) & $73.50 \pm 5.88$ \\
\hline Smoking (no. of smokers), n (\%) & $1(12.5 \%)$ \\
\hline Educational years & $15.25 \pm 4.71$ \\
\hline Marital status (no. of married/widowed), n (\%) & $4 / 4(50.0 \% / 50.0 \%)$ \\
\hline Medical history-Clinical evaluation & \\
\hline Diagnosis/drug treatment for hypertension, $\mathrm{n}(\%)$ & $5(62.5 \%)$ \\
\hline Diagnosis/drug treatment for dyslipidemia, n (\%) & $2(25.0 \%)$ \\
\hline Drug treatment, n (\%) & $6(75.0 \%)$ \\
\hline Dietary supplements, n (\%) & $3(37.5 \%)$ \\
\hline Evacuation frequency (times $\cdot \mathrm{d}^{-1}$ ) & $1.00(1.00-1.00)$ \\
\hline \multicolumn{2}{|l|}{ Anthropometric measurements } \\
\hline Body weight (kg) & $63.55(62.55-73.63)$ \\
\hline Height (m) & $1.64 \pm 0.09$ \\
\hline $\mathrm{BMI}\left(\mathrm{kg} \cdot \mathrm{m}^{-2}\right)$ & $25.14 \pm 3.16$ \\
\hline \multicolumn{2}{|l|}{ Nutritional analysis-Physical activity } \\
\hline Energy intake $\left(\mathrm{kcal} \cdot \mathrm{d}^{-1}\right)$ & $1585.99 \pm 582.31$ \\
\hline Carbohydrate (\% of energy) & $42.91 \pm 6.68$ \\
\hline Carbohydrate $\left(\mathrm{g} \cdot \mathrm{d}^{-1}\right)$ & $173.99 \pm 69.47$ \\
\hline Protein (\% of energy) & $18.10 \pm 4.24$ \\
\hline Protein $\left(g \cdot \mathrm{d}^{-1}\right)$ & $69.83 \pm 20.89$ \\
\hline Fat (\% of energy) & $36.94 \pm 5.54$ \\
\hline
\end{tabular}


Table 2. Cont.

\begin{tabular}{cc}
\hline \multicolumn{2}{c}{ Sociodemographic Parameters } \\
\hline Fat $\left(\mathrm{g} \cdot \mathrm{d}^{-1}\right)$ & $67.38 \pm 30.73$ \\
SFA $\left(\mathrm{g} \cdot \mathrm{d}^{-1}\right)$ & $22.41 \pm 9.60$ \\
MUFA $\left(\mathrm{g} \cdot \mathrm{d}^{-1}\right)$ & $22.83(19.78-37.70)$ \\
PUFA $\left(\mathrm{g} \cdot \mathrm{d}^{-1}\right)$ & $8.52(5.20-9.72)$ \\
Fiber $\left(\mathrm{g} \cdot \mathrm{d}^{-1}\right)$ & $14.89 \pm 10.26$ \\
Total Physical Activity $\left(\mathrm{MET}-\mathrm{min} \cdot \mathrm{wk}^{-1}\right)$ & $1333.38 \pm 876.79$ \\
Sitting or resting time $\left(\mathrm{h} \cdot \mathrm{wk}^{-1}\right)$ & $34.13 \pm 14.21$ \\
Moderate level of physical activity, $\mathrm{n}(\%)$ & $6(75.0 \%)$ \\
\hline
\end{tabular}

Values are expressed as mean and SD for parametric or median and Q1-Q3 quartiles for nonparametric data; BMI: Body Mass Index; MET: Metabolic equivalent of task; MUFA: Monounsaturated Fatty Acids; PUFA: Polyunsaturated Fatty Acids; SFA: Saturated Fatty Acids.

\subsection{Gut Microbiota Analysis}

At the baseline $(t=0 \mathrm{~h})$, levels of total bacteria, butyrate producers and $C$. perfringens had significant differences compared to NC and/or INU2, especially in the case of PEWS and CC2WS. Nevertheless, similar initial levels of bifidobacteria, lactobacilli and Bacteroides spp. were detected in all mushrooms, compared to negative and positive controls (Table 3).

After 24-h fermentation, total bacterial levels significantly increased in nearly all substrates compared to NC (Table 3). Positive control (INU2) induced both a significant bifidogenic and lactogenic effect, compared to NC and baseline $(t=0 \mathrm{~h})$. Nevertheless, mushrooms had a more versatile role, with $P$. eryngii inducing a strong lactogenic effect whereas representatives of $P$. ostreatus and C. cylindracea demonstrated a significant bifidogenic effect. Indeed, these effects were significant compared to NC and quite analogous to that of the prebiotic inulin after $24-\mathrm{h}$ fermentation. Furthermore, paired-samples analysis revealed that CC2WS induced significant increase in both baseline lactobacilli and bifidobacterial levels, whereas initial bifidobacterial levels were also elevated after the fermentation with mushrooms from the POWS, POLWS, PEWS, PEWSGM and HEOLRP treatments.

Bacteroides spp. levels were reduced in the case of C. cylindracea mushrooms after 24-h fermentation, likewise NC. On the contrary, the effect of all other tested mushroom was similar to the effect of inulin on Bacteroides population, with stable or rather increased levels after 24-h fermentation. Clostridial levels increased in the case of PEWS, POOLRP and CC2WS, compared to baseline, whereas only POLWS had significant higher clostridial levels compared to NC after 24-h fermentation.

Butyrate producers (e.g., Faecalibacterium prausnitzii, Roseburia spp.-Eubacterium rectale) were significantly reduced under no carbohydrate source (NC), whereas, in the case of inulin (INU2), the levels of F. prausnitzii increased and levels of Roseburia spp.-E. rectale decreased. Based on our experimental data, 24-h fermentation of PEWSGM significantly increased the levels of both bacterial groups, while mushrooms deriving from several substrates had positive effects in F. prausnitzii population (e.g., POWS, POOLRP, POLWS, PEWS, CC505WS). Finally, in the case of HEOLRP, the levels of both butyrate producers were increased compared to NC after 24-h fermentation, though they remained rather stable compared to baseline data $(\mathrm{t}=0 \mathrm{~h})$.

Based on data from Table 3, we observed that P. ostreatus mushrooms cultivated in wheat straw (POWS, POLWS) were characterized by an increase in levels of bifidobacteria and F. prausnitzii. Furthermore, fermentation of $P$. eryngii and C. cylindracea mushrooms induced diverse effects in microbial profiling, irrespectively of strain (CC2WS, CC505WS) or cultivation substrate (PEWS, PEWSGM) used, whereas limited effects on the tested microorganisms were observed in the case of H. erinaceum treatments. In addition, the comparison of the same strain in different substrates (POWS vs. POOLRP, POLWS vs. POLTPOMW, PEWS vs. PEWSGM, HEBS vs. HEOLRP) or of different strains cultivated in the same substrate (POWS vs. POLWS, CC2WS vs. CC505WS) revealed only a significant 0.4-log mean difference in Roseburia sp.-E. rectale levels after 24-h fermentation of PEWS, compared to PEWSGM (8.07 \pm 0.28 vs. $8.43 \pm 0.26 \log _{10} 16 \mathrm{~S}$ copies, respectively, $\left.p=0.021\right)$. 
Table 3. Fecal microbial quantification (quantitative PCR (qPCR); $\log _{10}$ copies of $16 \mathrm{~S}$ rRNA gene $\mathrm{mL}^{-1}$ of sample) at baseline $(\mathrm{t}=0 \mathrm{~h})$ and after $24 \mathrm{~h}$ fermentation .

\begin{tabular}{|c|c|c|c|c|c|c|c|}
\hline \multicolumn{8}{|c|}{ Baseline $(t=0 \mathrm{~h})$} \\
\hline & Total Bacteria & $\begin{array}{l}\text { Lactobacillus } \\
\text { Group }\end{array}$ & Bifidobacterium spp. & Bacteroides spp. & $\begin{array}{l}\text { Clostridium } \\
\text { perfringens } \\
\text { group }\end{array}$ & $\begin{array}{c}\text { Roseburia } \\
\text { spp.-Eubacterium rectale }\end{array}$ & $\begin{array}{c}\text { Faecalibacterium } \\
\text { prausnitzii }\end{array}$ \\
\hline $\mathrm{NC}$ & $\begin{array}{c}10.11 \\
(10.02-10.21)\end{array}$ & $\begin{array}{c}6.13 \\
(5.84-7.20)\end{array}$ & $\begin{array}{c}8.79 \\
(7.40-9.07)\end{array}$ & $9.48 \pm 0.27$ & $\begin{array}{c}6.59 \\
(6.35-6.75)\end{array}$ & $\begin{array}{c}8.45 \\
(8.17-8.78)\end{array}$ & $\begin{array}{c}8.86 \\
(8.60-9.06)\end{array}$ \\
\hline INU2 & $\begin{array}{c}10.20 \\
(10.08-10.30)\end{array}$ & $\begin{array}{c}6.19 \\
(5.84-7.32)\end{array}$ & $\begin{array}{c}8.81 \\
(7.38-9.04)\end{array}$ & $9.56 \pm 0.24$ & $\begin{array}{c}6.62 \\
(6.44-6.83)\end{array}$ & $\begin{array}{c}8.53 \\
(8.22-8.72)\end{array}$ & $\begin{array}{c}8.97 \\
(8.59-9.05)\end{array}$ \\
\hline POWS & $\begin{array}{c}9.99^{+} \\
(9.94-10.12)\end{array}$ & $\begin{array}{c}6.09 \\
(5.82-7.20)\end{array}$ & $\begin{array}{c}8.78 \\
(7.31-9.02)\end{array}$ & $9.50 \pm 0.21$ & $\begin{array}{c}6.44 \\
(6.11-6.69)\end{array}$ & $\begin{array}{c}8.20 \\
(8.04-8.55)\end{array}$ & $\begin{array}{c}8.76 \\
(8.40-8.86)\end{array}$ \\
\hline POOLRP & $\begin{array}{c}9.96^{*,+} \\
(9.81-10.01)\end{array}$ & $\begin{array}{c}5.98 \\
(5.73-7.06)\end{array}$ & $\begin{array}{c}8.71 \\
(7.28-9.06)\end{array}$ & $9.40 \pm 0.17$ & $\begin{array}{c}6.36 \\
(6.08-6.84)\end{array}$ & $\begin{array}{c}8.15 t \\
(8.05-8.49)\end{array}$ & $\begin{array}{c}8.67 \\
(8.40-8.82)\end{array}$ \\
\hline POLWS & $\begin{array}{c}10.14 \\
(10.06-10.22)\end{array}$ & $\begin{array}{c}6.12 \\
(5.84-7.26)\end{array}$ & $\begin{array}{c}8.74 \\
(7.25-9.08)\end{array}$ & $9.59 \pm 0.18$ & $\begin{array}{c}6.76 \\
(6.51-6.91)\end{array}$ & $\begin{array}{c}8.58 \\
(8.26-8.74)\end{array}$ & $\begin{array}{c}9.00 \\
(8.64-9.13)\end{array}$ \\
\hline POLTPOMW & $\begin{array}{c}10.02 \\
(9.89-10.15)\end{array}$ & $\begin{array}{c}6.68 \\
(6.24-7.28)\end{array}$ & $\begin{array}{c}7.87 \\
(6.80-8.87)\end{array}$ & $9.64 \pm 0.31$ & $\begin{array}{c}6.86 \\
(6.34-7.13)\end{array}$ & $\begin{array}{c}8.60 \\
(8.57-8.61)\end{array}$ & $\begin{array}{c}8.79 \\
(8.64-9.13)\end{array}$ \\
\hline PEWS & $\begin{array}{c}9.84^{*, \dagger} \\
(9.67-9.88)\end{array}$ & $\begin{array}{c}5.94 \\
(5.60-7.04)\end{array}$ & $\begin{array}{c}8.73 \\
(7.29-8.97)\end{array}$ & $9.37 \pm 0.22$ & $\begin{array}{c}6.24 \\
(5.97-6.73)\end{array}$ & $\begin{array}{c}8.00^{*,+} \\
(7.72-8.23)\end{array}$ & $\begin{array}{c}8.53^{*, \dagger} \\
(8.35-8.74)\end{array}$ \\
\hline PEWSGM & $\begin{array}{c}10.05^{\dagger} \\
(9.72-10.09)\end{array}$ & $\begin{array}{c}6.03 \\
(5.72-7.21)\end{array}$ & $\begin{array}{c}8.71 \\
(6.98-9.10)\end{array}$ & $9.51 \pm 0.23$ & $\begin{array}{c}6.48 \\
(6.21-6.76)\end{array}$ & $\begin{array}{c}8.08^{*, \dagger} \\
(7.96-8.51)\end{array}$ & $\begin{array}{c}8.74 \\
(8.42-8.87)\end{array}$ \\
\hline HEBS & $\begin{array}{c}10.10 \\
(9.97-10.30)\end{array}$ & $\begin{array}{c}6.46 \\
(5.83-7.20)\end{array}$ & $\begin{array}{c}7.90 \\
(6.74-9.02)\end{array}$ & $9.45 \pm 0.22$ & $\begin{array}{c}6.11^{*,+} \\
(5.73-6.40)\end{array}$ & $\begin{array}{c}8.32 \\
(8.04-8.51)\end{array}$ & $\begin{array}{c}8.70 \\
(8.39-8.87)\end{array}$ \\
\hline HEOLRP & $\begin{array}{c}10.12 \\
(10.02-10.18)\end{array}$ & $\begin{array}{c}5.99 \\
(5.74-7.21)\end{array}$ & $\begin{array}{c}8.87 \\
(7.54-9.15)\end{array}$ & $9.51 \pm 0.29$ & $\begin{array}{c}6.36 \\
(5.96-6.65)\end{array}$ & $\begin{array}{c}8.33 \\
(8.12-8.50)\end{array}$ & $\begin{array}{c}8.84 \\
(8.66-8.98)\end{array}$ \\
\hline CC2WS & $\begin{array}{c}9.89 *,+ \\
(9.79-9.96)\end{array}$ & $\begin{array}{c}5.92 \\
(5.43-6.60)\end{array}$ & $\begin{array}{c}8.36 \\
(7.06-8.92)\end{array}$ & $9.32 \pm 0.28$ & $\begin{array}{c}5.83^{*,+} \\
(5.22-6.12)\end{array}$ & $\begin{array}{c}8.09 *,+ \\
(7.82-8.38)\end{array}$ & $\begin{array}{c}8.52^{*,+} \\
(8.27-8.74)\end{array}$ \\
\hline CC505WS & $\begin{array}{c}10.11^{+} \\
(9.81-10.15)\end{array}$ & $\begin{array}{c}5.98 \\
(5.61-6.89)\end{array}$ & $\begin{array}{c}8.53 \\
(7.26-9.06)\end{array}$ & $9.47 \pm 0.30$ & $\begin{array}{c}6.12^{*,+} \\
(5.66-6.49)\end{array}$ & $\begin{array}{c}8.53 \\
(7.26-9.06)\end{array}$ & $\begin{array}{c}8.30 \\
(8.19-8.62)\end{array}$ \\
\hline \multicolumn{8}{|c|}{ 24-h Fermentation $(t=24 h)$} \\
\hline & Total Bacteria & Lactobacillus Group & Bifidobacterium spp. & Bacteroides spp. & $\begin{array}{c}\text { Clostridium } \\
\text { perfringens Group }\end{array}$ & $\begin{array}{c}\text { Roseburia } \\
\text { spp.-Eubacterium rectale }\end{array}$ & $\begin{array}{c}\text { Faecalibacterium } \\
\text { prausnitzii }\end{array}$ \\
\hline $\mathrm{NC}$ & $\begin{array}{c}9.98^{+, \mathrm{a}} \\
(9.86-10.06)\end{array}$ & $\begin{array}{c}5.95^{\dagger} \\
(5.69-7.05)\end{array}$ & $\begin{array}{c}8.73^{\dagger} \\
(7.34-9.16)\end{array}$ & $9.04 \pm 0.38^{t, a}$ & $\begin{array}{c}6.38 \\
(6.03-6.69)\end{array}$ & $\begin{array}{c}7.97^{\mathrm{a}} \\
(7.09-8.15)\end{array}$ & $\begin{array}{c}8.29^{\dagger, \mathrm{a}} \\
(7.83-8.62)\end{array}$ \\
\hline INU2 & $\begin{array}{c}10.28^{*} \\
(10.14-10.32)\end{array}$ & $\begin{array}{c}7.66^{*, \mathrm{a}} \\
(7.03-9.00)\end{array}$ & $\begin{array}{c}9.83^{*, \mathrm{a}} \\
(8.11-9.96)\end{array}$ & $9.68 \pm 0.23 *$ & $\begin{array}{c}6.42 \\
(6.22-6.70)\end{array}$ & $\begin{array}{c}8.15^{\mathrm{a}} \\
(7.91-8.50)\end{array}$ & $\begin{array}{c}9.06^{*, \mathrm{a}} \\
(8.66-9.15)\end{array}$ \\
\hline POWS & $\begin{array}{c}10.36^{*, a} \\
(10.14-10.40)\end{array}$ & $\begin{array}{c}6.48 \\
(5.87-7.59)\end{array}$ & $\begin{array}{c}9.49^{\mathrm{a}} \\
(8.12-9.66)\end{array}$ & $9.53 \pm 0.33$ * & $\begin{array}{c}6.58 \\
(6.25-6.92)\end{array}$ & $\begin{array}{c}8.38^{*} \\
(7.97-8.46)\end{array}$ & $\begin{array}{c}9.06^{*, a} \\
(8.78-9.39)\end{array}$ \\
\hline
\end{tabular}


Table 3. Cont.

\begin{tabular}{|c|c|c|c|c|c|c|c|}
\hline \multicolumn{8}{|c|}{ Baseline $(t=0 \mathrm{~h})$} \\
\hline POOLRP & $\begin{array}{c}10.38^{*, \mathrm{a}} \\
(10.20-10.49)\end{array}$ & $\begin{array}{c}6.56 \\
(5.84-7.35)\end{array}$ & $\begin{array}{c}9.49^{*, \mathrm{a}} \\
(8.36-9.82)\end{array}$ & $9.61 \pm 0.16^{*, a}$ & $\begin{array}{c}6.71^{\mathrm{a}} \\
(6.60-6.82)\end{array}$ & $\begin{array}{c}8.33 * \\
(8.01-8.47)\end{array}$ & $\begin{array}{c}9.10 *, \mathrm{a} \\
(8.97-9.32)\end{array}$ \\
\hline POLWS & $\begin{array}{c}10.39^{*, a} \\
(10.28-10.46)\end{array}$ & $\begin{array}{c}6.59 \\
(5.87-8.26)\end{array}$ & $\begin{array}{c}9.55^{\mathrm{a}} \\
(8.27-9.66)\end{array}$ & $9.62 \pm 0.34$ * & $\begin{array}{c}6.75 * \\
(6.57-7.32)\end{array}$ & $\begin{array}{c}8.50 *,+ \\
(8.40-8.79)\end{array}$ & $\begin{array}{c}9.21 *, \mathrm{a} \\
(8.96-9.32)\end{array}$ \\
\hline POLTPOMW & $\begin{array}{c}10.13 \\
(10.08-10.36)\end{array}$ & $\begin{array}{c}7.53 \\
(6.09-8.83)\end{array}$ & $\begin{array}{c}8.65 \\
(6.99-9.57)\end{array}$ & $9.46 \pm 0.47$ * & $\begin{array}{c}6.50 \\
(6.44-6.74)\end{array}$ & $\begin{array}{c}8.58^{*, \dagger} \\
(8.34-8.72)\end{array}$ & $\begin{array}{c}8.78 \\
(8.54-9.03)\end{array}$ \\
\hline PEWS & $\begin{array}{c}10.29^{*, \mathrm{a}} \\
(10.18-10.37)\end{array}$ & $\begin{array}{c}8.58^{*, \mathrm{a}} \\
(7.40-9.32)\end{array}$ & $\begin{array}{c}9.29^{\mathrm{a}} \\
(8.08-9.54)\end{array}$ & $9.50 \pm 0.32^{*}$ & $\begin{array}{c}6.52^{\mathrm{a}} \\
(6.13-6.91)\end{array}$ & $\begin{array}{c}7.99^{\mathrm{a}} \\
(7.89-8.33)\end{array}$ & $\begin{array}{c}9.02 *, \mathrm{a} \\
(8.75-9.58)\end{array}$ \\
\hline PEWSGM & $\begin{array}{c}10.44^{*,+, a} \\
(10.30-10.50)\end{array}$ & $\begin{array}{c}7.93 * \text { *a } \\
(6.81-9.21)\end{array}$ & $\begin{array}{c}9.36^{\mathrm{a}} \\
(8.29-9.74)\end{array}$ & $9.69 \pm 0.23^{*}$ & $\begin{array}{c}6.72 \\
(6.28-7.08)\end{array}$ & $\begin{array}{c}8.51^{*,+, a} \\
(8.39-8.58)\end{array}$ & $\begin{array}{c}9.18^{*, \mathrm{a}} \\
(8.96-9.79)\end{array}$ \\
\hline HEBS & $\begin{array}{c}10.12 \\
(9.78-10.18)\end{array}$ & $\begin{array}{c}6.45 \\
(5.86-7.97)\end{array}$ & $\begin{array}{c}8.19^{+} \\
(6.72-9.29)\end{array}$ & $9.54 \pm 0.27^{*}$ & $\begin{array}{c}6.09 \\
(5.89-6.66)\end{array}$ & $\begin{array}{c}8.01 \\
(7.43-8.25)\end{array}$ & $\begin{array}{c}8.49 \\
(8.02-8.97)\end{array}$ \\
\hline HEOLRP & $\begin{array}{c}10.19 * \\
(10.10-10.36)\end{array}$ & $\begin{array}{c}6.29^{\dagger} \\
(5.72-7.14)\end{array}$ & $\begin{array}{c}9.13^{+, \mathrm{a}} \\
(7.60-9.47)\end{array}$ & $9.61 \pm 0.27^{*}$ & $\begin{array}{c}6.50 \\
(6.18-6.80)\end{array}$ & $\begin{array}{c}8.34 * \\
(8.06-8.42)\end{array}$ & $\begin{array}{c}8.79 * \\
(8.47-9.15)\end{array}$ \\
\hline CC2WS & $\begin{array}{c}10.14^{\mathrm{a}} \\
(9.96-10.24)\end{array}$ & $\begin{array}{c}6.23^{\mathrm{a}} \\
(5.87-9.14)\end{array}$ & $\begin{array}{c}9.38^{\mathrm{a}} \\
(7.64-9.88)\end{array}$ & $8.88 \pm 0.45^{t, a}$ & $\begin{array}{c}6.37^{\mathrm{a}} \\
(5.67-6.52)\end{array}$ & $\begin{array}{c}7.85^{+, \mathrm{a}} \\
(7.52-8.14)\end{array}$ & $\begin{array}{c}8.74^{\mathrm{a}} \\
(8.48-9.00)\end{array}$ \\
\hline CC505WS & $\begin{array}{c}10.25^{*, \mathrm{a}} \\
(10.07-10.35)\end{array}$ & $\begin{array}{c}6.27 \\
(5.75-9.22)\end{array}$ & $\begin{array}{c}9.69^{*, \mathrm{a}} \\
(7.84-9.83)\end{array}$ & $9.07 \pm 0.52^{\dagger, a}$ & $\begin{array}{c}6.40 \\
(5.92-6.62)\end{array}$ & $\begin{array}{c}8.07 \\
(7.90-8.25)\end{array}$ & $\begin{array}{c}8.98^{*, \mathrm{a}} \\
(9.00-9.24)\end{array}$ \\
\hline$P$ overall & 0.018 & 0.733 & 0.939 & 0.001 & 0.022 & 0.001 & 0.143 \\
\hline
\end{tabular}

Values are expressed as mean and SD for parametric or median and Q1-Q3 quartiles for nonparametric data (for POLTPOMW and HEBS $\mathrm{n}=4$ - for CC2WS and CC505 $\mathrm{n}=7$; * significant difference compared to NC $(p<0.05)$ at baseline $(t=0 \mathrm{~h})$ or after 24-h fermentation ( $\mathrm{t}=24 \mathrm{~h}$ ) (repeated measures ANOVA after Bonferroni's adjustment for multiplicity or Friedman's test)

t: significant difference compared to INU2 $(p<0.05)$ at baseline $(t=0 h)$ or after 24-h fermentation $(t=24 \mathrm{~h})$ (repeated measures ANOVA after Bonferroni's adjustment for multiplicity or Friedman's test); a: significant difference compared to baseline (paired-samples T test for parametric data and Wilcoxon signed ranks test for non-parametric data). 


\subsection{Prebiotic Indexes}

Inulin and tested mushrooms demonstrated positive mean PIs after 24-h fermentation, with higher levels detected in the case of the prebiotic inulin and P. eryngii (PEWS, PEWSGM) and C. cylindracea (CC2WS, CC505WS) mushrooms (overall $p=0.081)$. Furthermore, only INU2 $(p=0.005)$ and PEWS $(p=0.021)$ resulted in significantly higher PIs compared to NC after $24-\mathrm{h}$ fermentation (Figure 1a,b). It is worth mentioning that there was no difference between INU2 and PEWS ( $p=0.401)$. In addition, only inulin and PEWS induced positive PIs results in all eight runs of the experiment with different fecal donors (Supplementary Materials, Table S1).



Figure 1. Prebiotic Indexes (PIs) of the tested mushrooms and controls after 24-h fermentation (a: NC, HEBS, HEOLRP, POOLRP, POWS, POLTPOMW and POLWS treatments; $\mathbf{b}$ : INU2, PEWSGM, PEWS, CC2WS and CC505WS treatments);Values are expressed as mean and SD (for POLTPOMW and HEBS $\mathrm{n}=4$-for CC2WS and CC505 n = 7); *: significant difference compared to NC $(p<0.05)$ (Mann-Whitney test); + : significant difference compared to INU2 ( $p<0.05)$ (Mann-Whitney test).

Though no significant overall differences were observed among PIs for the first four runs of the experiment with all substrates available $(p=0.717)$, once again INU2 $(p=0.149)$, PEWS $(p=0.149)$ and CC2WS ( $p=0.149$ ) resulted in considerably higher PIs compared to NC (Supplementary Materials, Figure S1a,b). Further analysis in mean PIs values of mushrooms indicated also no significant strainor substrate-specific differentiation [e.g., POWS vs. POOLRP, $p=1.000$; POLWS vs. POLTPOMW, $p=0.062(p=1.000$ for $\mathrm{n}=4)$; POWS vs. POLWS, $p=0.753$; PEWS vs. PEWSGM, $p=0.674$; HEBS vs. HEOLRP, $p=0.089$ ( $p=0.683$ for $n=4)$; CC2WS vs. CC505WS, $p=0.406$ ]. Finally, it was noticed that prebiotic potential exhibited quite high variability among the eight different fecal donors (Supplementary Materials, Table S1).

Statistical analysis revealed significant positive correlations of mean PIs values of mushrooms with their average total glucan (Spearman's rho 0.806, $p=0.005$ ) and $\beta$-glucan content (Spearman's rho 0.758, $p=0.011$ ) for all the available data and for the first four runs of the fermentation procedure (total glucan: Spearman's rho 0.758, $p=0.011$; $\beta$-glucan: Spearman's rho 0.709, $p=0.022$ ) (Supplementary Materials, Figure S2a-d). No significant correlations were detected in terms of $\alpha$-glucan content.

\subsection{Short Chain Fatty Acids (SCFAs) Analysis}

Concentrations of total volatile fatty acids (VFAs) and SCFAs, so as molar ratios of SCFAs, at baseline and after 8-h and 24-h fermentation are presented in Tables 4 and 5. Furthermore, Figures $2 \mathrm{a}-\mathrm{d}$ and $3 \mathrm{a}, \mathrm{b}$ illustrate the mean differences of total VFAs ( $\triangle$ TVFAs) and SCFAs concentration after $8 \mathrm{~h}$ and $24 \mathrm{~h}$ of fermentation. 




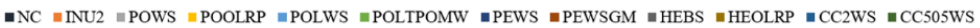



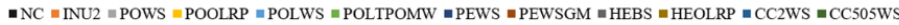

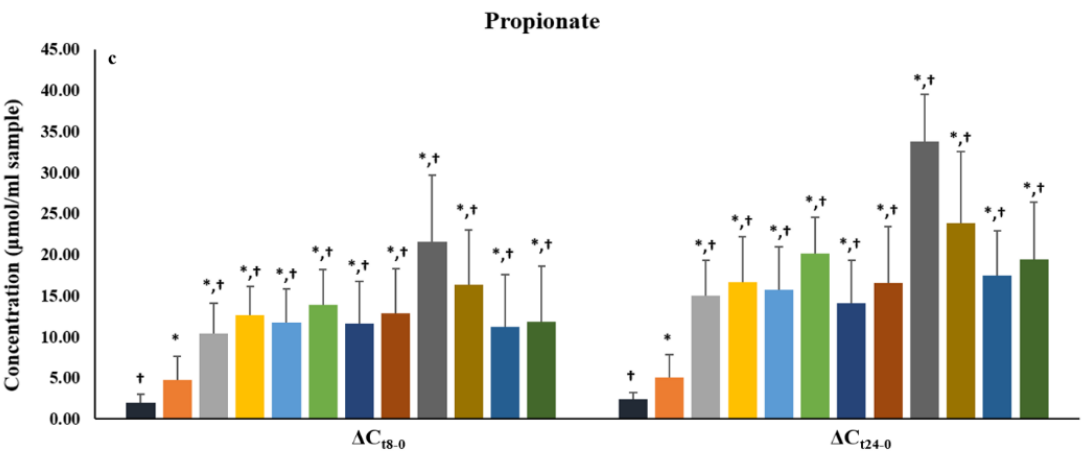

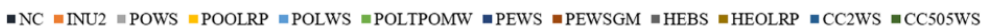

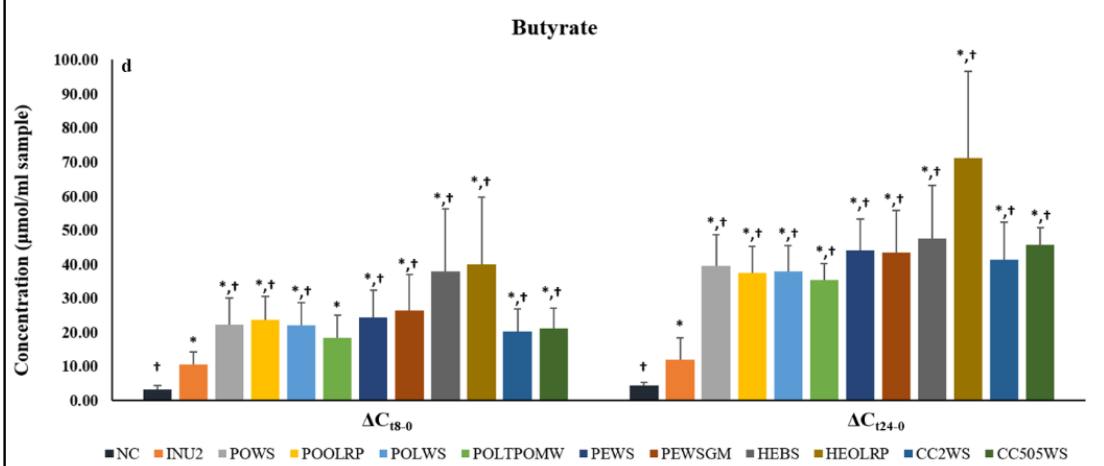

Figure 2. Differences $(\Delta)$ in concentrations $\left(\mu \mathrm{mol} \mathrm{mL}^{-1}\right.$ ) of (a) total volatile fatty acids (VFAs), (b) acetate, (c) propionate and (d) butyrate after 8-h fermentation ( $\Delta$ Ct8-0) and 24-h fermentation $(\Delta \mathrm{Ct} 24-0)$ compared to baseline. Values are expressed as mean and $\mathrm{SD}$, where $\Delta \mathrm{Ct} 8-0$ is defined as 'Concentration $\mathrm{t}=8 \mathrm{~h}$ minus Concentration $\mathrm{t}=0 \mathrm{~h}$ ' and $\Delta \mathrm{Ct} 24-0$ is defined as 'Concentration $\mathrm{t}=24 \mathrm{~h}$ minus Concentration $\mathrm{t}=0$ h' (for POLTPOMW and HEBS $\mathrm{n}=4$-for CC2WS and CC505 $\mathrm{n}=7$ ); *: significant difference compared to NC $(p<0.05)$ (Mann-Whitney test or $t$-test); + : significant difference compared to INU2 $(p<0.05)$ (Mann-Whitney test or $t$-test). 


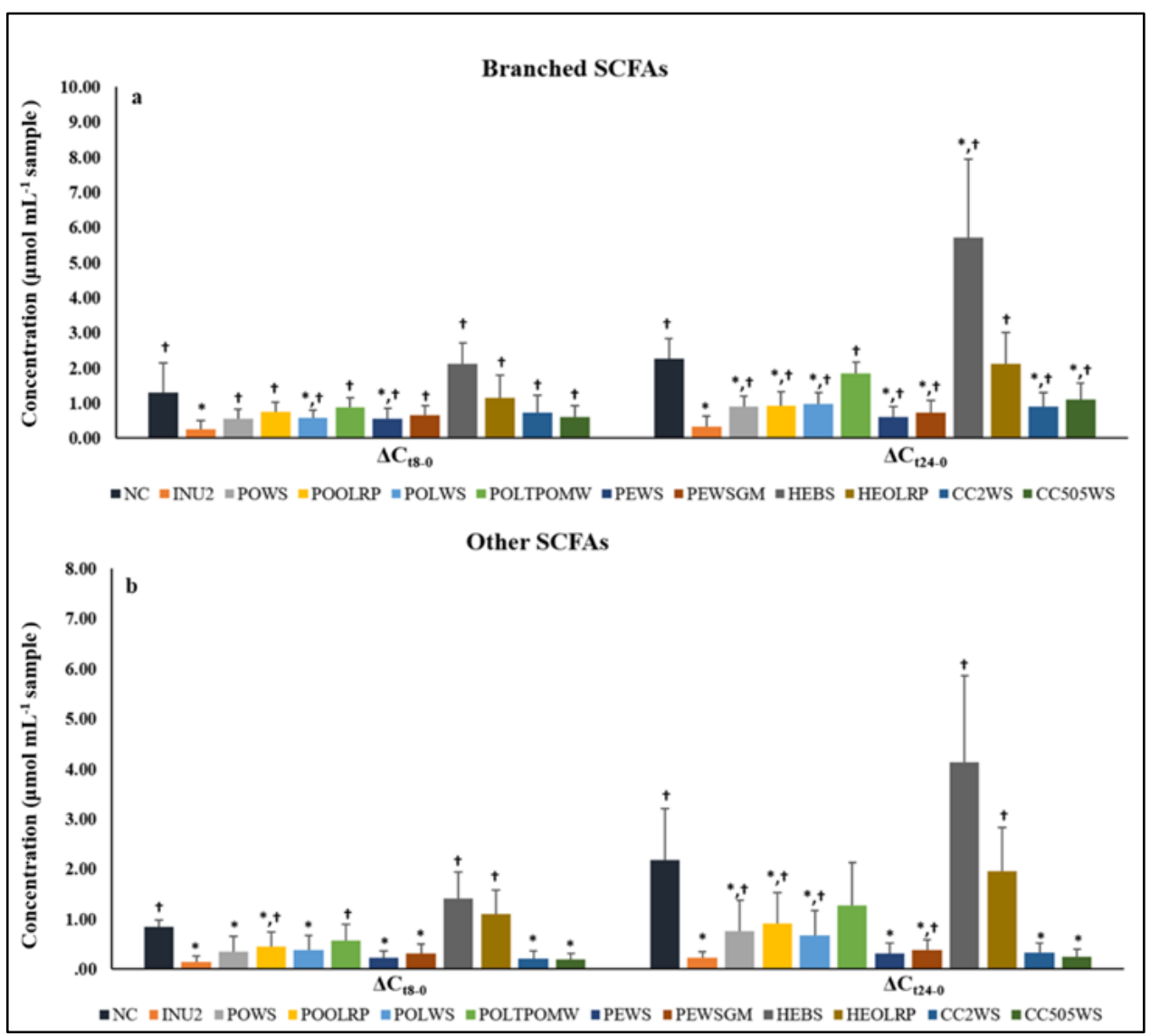

Figure 3. Differences $(\Delta)$ in concentrations $\left(\mu \mathrm{mol} \mathrm{mL} \mathrm{m}^{-1}\right)$ of $(\mathbf{a})$ branched SCFAs (BSFAs) and (b) other SCFAs after 8-h fermentation ( $\Delta \mathrm{Ct} 8-0)$ and 24 -h fermentation ( $\Delta \mathrm{Ct} 24-0)$ compared to baseline. Values are expressed as mean and SD, where $\Delta \mathrm{Ct} 8-0$ is defined as 'Concentration $\mathrm{t}=8 \mathrm{~h}$ minus Concentration $\mathrm{t}=0 \mathrm{~h}$ ' and $\Delta \mathrm{Ct} 24-0$ is defined as 'Concentration $\mathrm{t}=24 \mathrm{~h}$ minus Concentration $\mathrm{t}=0 \mathrm{~h}$ ' (for POLTPOMW and HEBS $\mathrm{n}=4$ - for CC2WS and CC505 n = 7); *: significant difference compared to NC $(p<0.05)$ (Mann-Whitney test or $t$-test); + : significant difference compared to INU2 $(p<0.05)$ (Mann-Whitney test or $t$-test).

Table 4. Total volatile fatty acids (VFAs) and SCFAs concentrations ( $\mu \mathrm{mol} \mathrm{mL}-1$ of sample) at baseline and after 8-h and 24-h fermentation.

\begin{tabular}{|c|c|c|c|c|c|c|}
\hline \multicolumn{7}{|c|}{$\begin{array}{c}\text { Baseline }(t=0 \mathrm{~h}) \\
\text { Concentrations }\left(\mu \mathrm{mol} \mathrm{mL}^{-1}\right)\end{array}$} \\
\hline & Total VFAs & Acetate & Propionate & Butyrate & BSCFAs & Other SCFAs \\
\hline NC & $3.80 \pm 1.33^{+}$ & $\begin{array}{c}1.55 \\
(1.32-2.14)\end{array}$ & $\begin{array}{c}0.43 \\
(0.30-0.59)\end{array}$ & $\begin{array}{c}1.14^{\dagger} \\
(0.61-2.15)\end{array}$ & $\begin{array}{c}0.10 \\
(0.06-0.16)\end{array}$ & $\begin{array}{c}0.11 \\
(0.09-0.22)\end{array}$ \\
\hline INU2 & $2.59 \pm 0.71$ * & $\begin{array}{c}1.49 \\
(0.81-1.65)\end{array}$ & $\begin{array}{c}0.37 \\
(0.21-0.45)\end{array}$ & $\begin{array}{c}0.67^{*} \\
(0.58-0.83)\end{array}$ & $\begin{array}{c}0.10 \\
(0.09-0.11)\end{array}$ & $\begin{array}{c}0.10 \\
(0.08-0.16)\end{array}$ \\
\hline POWS & $3.12 \pm 0.68$ & $\begin{array}{c}1.56 \\
(1.13-1.69)\end{array}$ & $\begin{array}{c}0.32 \\
(0.16-0.53)\end{array}$ & $\begin{array}{c}1.14^{\dagger} \\
(0.87-1.37)\end{array}$ & $\begin{array}{c}0.07^{\dagger} \\
(0.06-0.08)\end{array}$ & $\begin{array}{c}0.10 \\
(0.09-0.20)\end{array}$ \\
\hline POOLRP & $2.92 \pm 0.68 *$ & $\begin{array}{c}1.48 \\
1.05-1.80)\end{array}$ & $\begin{array}{c}0.24 * \\
(0.16-0.39)\end{array}$ & $\begin{array}{c}0.97^{+} \\
(0.90-1.26)\end{array}$ & $\begin{array}{c}0.07^{+} \\
(0.06-0.09)\end{array}$ & $\begin{array}{c}0.11 \\
(0.09-0.14)\end{array}$ \\
\hline POLWS & $2.90 \pm 0.97 *$ & $\begin{array}{c}1.44 \\
(0.75-1.84)\end{array}$ & $\begin{array}{c}0.26 \\
(0.16-0.47)\end{array}$ & $\begin{array}{c}0.99^{+} \\
(0.82-1.36)\end{array}$ & $\begin{array}{c}0.07^{\dagger} \\
(0.05-0.08)\end{array}$ & $\begin{array}{c}0.10 \\
(0.08-0.14)\end{array}$ \\
\hline POLTPOMW & $2.94 \pm 0.52$ & $\begin{array}{c}1.60 \\
(1.24-1.80)\end{array}$ & $\begin{array}{c}0.29 \\
(0.22-0.46)\end{array}$ & $\begin{array}{c}0.95 \\
(0.68-1.04)\end{array}$ & $\begin{array}{c}0.07^{+} \\
(0.06-0.10)\end{array}$ & $\begin{array}{c}0.10 \\
(0.09-0.13)\end{array}$ \\
\hline PEWS & $2.66 \pm 0.72 *$ & $\begin{array}{c}1.33 \\
(0.89-1.67)\end{array}$ & $\begin{array}{c}0.25 * \\
(0.18-0.39)\end{array}$ & $\begin{array}{c}0.90 \\
(0.63-0.99)\end{array}$ & $\begin{array}{c}0.08^{+} \\
(0.05-0.08)\end{array}$ & $\begin{array}{c}0.10 \\
(0.09-0.15)\end{array}$ \\
\hline
\end{tabular}


Table 4. Cont

\begin{tabular}{|c|c|c|c|c|c|c|}
\hline & & & & & & \\
\hline PEWSGM & $2.82 \pm 0.78$ & $\begin{array}{c}1.55 \\
(1.03-1.89)\end{array}$ & $\begin{array}{c}0.28 * \\
(0.19-0.41)\end{array}$ & $\begin{array}{c}0.90 \\
(0.66-0.98)\end{array}$ & $\begin{array}{c}0.07^{+} \\
(0.06-0.09)\end{array}$ & $\begin{array}{c}0.10 \\
(0.09-0.14)\end{array}$ \\
\hline HEBS & $2.90 \pm 0.62$ & $\begin{array}{c}1.52 \\
(1.19-1.90)\end{array}$ & $\begin{array}{c}0.32 \\
(0.21-0.48)\end{array}$ & $\begin{array}{c}0.88 \\
(0.66-0.95)\end{array}$ & $\begin{array}{c}0.08 \\
(0.06-0.11)\end{array}$ & $\begin{array}{c}0.12 \\
(0.08-0.15)\end{array}$ \\
\hline HEOLRP & $3.32 \pm 0.93$ & $\begin{array}{c}2.15^{+} \\
(1.36-2.68)\end{array}$ & $\begin{array}{c}0.26 \\
(0.21-0.42)\end{array}$ & $\begin{array}{c}0.86 \\
(0.62-0.93)\end{array}$ & $\begin{array}{c}0.07 \\
(0.06-0.09)\end{array}$ & $\begin{array}{c}0.09 \\
(0.08-0.12)\end{array}$ \\
\hline CC2WS & $2.80 \pm 0.75 *$ & $\begin{array}{c}1.48 \\
(1.00-1.83)\end{array}$ & $\begin{array}{c}0.28 \\
(0.21-0.48)\end{array}$ & $\begin{array}{c}0.83 \\
(0.51-1.06)\end{array}$ & $\begin{array}{c}0.08 \\
(0.06-0.09)\end{array}$ & $\begin{array}{c}0.11 \\
(0.08-0.15)\end{array}$ \\
\hline CC505WS & $2.60 \pm 0.62$ * & $\begin{array}{c}1.43 \\
(0.88-1.65)\end{array}$ & $\begin{array}{c}0.30 \\
(0.21-0.41)\end{array}$ & $\begin{array}{c}0.82 \\
(0.62-0.89)\end{array}$ & $\begin{array}{c}0.08 \\
(0.06-0.09)\end{array}$ & $\begin{array}{c}0.10 \\
(0.10-0.13)\end{array}$ \\
\hline \multicolumn{7}{|c|}{$\begin{array}{l}\text { 8-h Fermentation }(t=8 \mathrm{~h}) \\
\text { Concentrations }\left(\mu \mathrm{mol} \mathrm{mL}^{-1}\right)\end{array}$} \\
\hline & Total VFAs & Acetate & Propionate & Butyrate & BSCFAs & Other SCFAs \\
\hline $\mathrm{NC}$ & $\underset{t, \mathrm{a}}{20.32 \pm 4.57}$ & $\begin{array}{c}11.31^{\mathrm{a}} \\
(8.40-13.29)\end{array}$ & $\begin{array}{c}1.96^{\mathrm{a}} \\
(1.71-3.69)\end{array}$ & $\begin{array}{c}4.38^{\mathrm{a}} \\
(3.87-5.35)\end{array}$ & $\begin{array}{c}1.27^{+, \mathrm{a}} \\
(0.66-2.25)\end{array}$ & $\begin{array}{c}0.98^{+, \mathrm{a}} \\
(0.87-1.15)\end{array}$ \\
\hline INU2 & $\underset{*, \mathrm{a}}{41.77 \pm 12.51}$ & $\begin{array}{c}22.41^{\mathrm{a}} \\
(19.08-27.55)\end{array}$ & $\begin{array}{c}4.15^{\mathrm{a}} \\
(3.02-6.26)\end{array}$ & $\begin{array}{c}11.01^{\mathrm{a}} \\
(8.67-13.90)\end{array}$ & $\begin{array}{c}0.25^{*, \mathrm{a}} \\
(0.21-0.44)\end{array}$ & $\begin{array}{c}0.29 * \text { a } \\
(0.17-0.36)\end{array}$ \\
\hline POWS & $\begin{array}{c}66.43 \pm 16.33 \\
*, \mathrm{t}, \mathrm{a}\end{array}$ & $\begin{array}{c}31.21 *, \mathrm{a} \\
(22.93-38.89)\end{array}$ & $\begin{array}{c}9.34 *,+, \mathrm{a} \\
(7.56-14.24)\end{array}$ & $\begin{array}{c}26.21 *,+, a \\
(17.20-28.04)\end{array}$ & $\begin{array}{c}0.62 *, \mathrm{a} \\
(0.36-0.83)\end{array}$ & $\begin{array}{c}0.41^{*, a} \\
(0.29-0.67)\end{array}$ \\
\hline POOLRP & $\underset{*, t, a}{77.53} \pm 13.90$ & $\begin{array}{c}36.13 *,+, a \\
(34.86-41.51)\end{array}$ & $\begin{array}{c}11.90 *,+, a \\
(9.84-16.50)\end{array}$ & $\begin{array}{c}26.40 *,+, a \\
(20.33-29.90)\end{array}$ & $\begin{array}{c}0.75^{+, a} \\
(0.69-0.89)\end{array}$ & $\begin{array}{c}0.54 *,+, a \\
(0.37-0.83)\end{array}$ \\
\hline POLWS & $\begin{array}{c}67.66 \pm 11.90 \\
*,+, a\end{array}$ & $\begin{array}{c}32.49 *, \mathrm{a} \\
(27.15-37.04)\end{array}$ & $\begin{array}{c}10.62^{*, t, a} \\
(9.90-13.66)\end{array}$ & $\begin{array}{c}25.00 *,+, a \\
(18.50-26.36)\end{array}$ & $\begin{array}{c}0.58^{*,+, a} \\
(0.51-0.78)\end{array}$ & $\begin{array}{c}0.47^{*,+, a} \\
(0.29-0.76)\end{array}$ \\
\hline POLTPOMW & $\begin{array}{c}71.10 \pm 12.02 \\
*,+, \mathrm{a}\end{array}$ & $\begin{array}{c}36.19 *,+, a \\
(33.44-38.13)\end{array}$ & $\begin{array}{c}12.77^{*,+, \mathrm{a}} \\
(11.06-18.89)\end{array}$ & $\begin{array}{c}21.24^{*, \mathrm{a}} \\
(12.47-24.28)\end{array}$ & $\begin{array}{c}0.86^{+, a} \\
(0.75-1.23)\end{array}$ & $\begin{array}{c}0.70^{+, \mathrm{a}} \\
(0.34-0.97)\end{array}$ \\
\hline PEWS & $\begin{array}{c}73.68 \pm 19.96 \\
*, t, \mathrm{a}\end{array}$ & $\begin{array}{c}35.19 *,+, a \\
(28.88-41.37)\end{array}$ & $\begin{array}{c}10.82 *,+, a \\
(7.54-17.92)\end{array}$ & $\begin{array}{c}25.59 *,+, a \\
(21.29-32.67)\end{array}$ & $\begin{array}{c}0.62 * \text {,a } \\
(0.36-0.79)\end{array}$ & $\begin{array}{c}0.35^{*, a} \\
(0.22-0.46)\end{array}$ \\
\hline PEWSGM & $\begin{array}{c}81.16 \pm 24.52 \\
*, t, \mathrm{a}\end{array}$ & $\begin{array}{c}36.69 *,+, a \\
(29.40-51.05)\end{array}$ & $\begin{array}{c}10.75^{*,+, a} \\
(9.20-18.32)\end{array}$ & $\begin{array}{c}28.51 *,+, a \\
(15.61-37.37)\end{array}$ & $\begin{array}{c}0.86^{+, a} \\
(0.42-0.89)\end{array}$ & $\begin{array}{c}0.40^{*}, \mathrm{a} \\
(0.28-0.58)\end{array}$ \\
\hline HEBS & $\begin{array}{c}95.81 \pm 21.96 \\
*, t, a\end{array}$ & $\begin{array}{c}32.40 * \text {,a } \\
(28.36-33.84)\end{array}$ & $\begin{array}{c}23.08^{*, t, \mathrm{a}} \\
(13.49-29.10)\end{array}$ & $\begin{array}{c}42.97^{*,+, a} \\
(19.49-53.54)\end{array}$ & $\begin{array}{c}2.11^{+, a} \\
(1.69-2.80)\end{array}$ & $\begin{array}{c}1.74^{+, \mathrm{a}} \\
(0.93-1.89)\end{array}$ \\
\hline HEOLRP & $\begin{array}{c}89.70 \pm 20.70 \\
*, t, \mathrm{a}\end{array}$ & $\begin{array}{c}31.84 * \text {, a } \\
(25.12-36.75)\end{array}$ & $\begin{array}{l}17.66 *,+, a \\
(9.47-23.41)\end{array}$ & $\begin{array}{c}36.14 *,+, a \\
(28.32-60.43)\end{array}$ & $\begin{array}{c}1.05^{+, a} \\
(0.70-1.46)\end{array}$ & $\begin{array}{c}1.25^{+, a} \\
(0.94-1.59)\end{array}$ \\
\hline CC2WS & $\begin{array}{c}68.56 \pm 16.26 \\
*, t, \mathrm{a}\end{array}$ & $\begin{array}{c}30.75 *,+, a \\
(28.73-43.57)\end{array}$ & $\begin{array}{c}8.63 *,+, a \\
(6.90-15.27)\end{array}$ & $\begin{array}{c}21.92 *,+, a \\
(16.14-26.49)\end{array}$ & $\begin{array}{c}0.55^{+, a} \\
(0.51-0.98)\end{array}$ & $\begin{array}{c}0.30^{*, \mathrm{a}} \\
(0.20-0.45)\end{array}$ \\
\hline CC505WS & $\underset{*, t, a}{68.12 \pm 13.63}$ & $\begin{array}{c}32.26^{*,+, a} \\
(27.92-38.39)\end{array}$ & $\begin{array}{c}8.73 *,+, \mathrm{a} \\
(7.52-14.72)\end{array}$ & $\begin{array}{c}21.82^{*, t, a} \\
(18.77-25.93)\end{array}$ & $\begin{array}{c}0.75^{*,+, a} \\
(0.42-0.80)\end{array}$ & $\begin{array}{c}0.26^{*, a} \\
(0.23-0.40)\end{array}$ \\
\hline \multicolumn{7}{|c|}{ 24-h Fermentation $(t=24 h)$} \\
\hline $\mathrm{NC}$ & $\begin{array}{c}\text { Total VFAs } \\
27.41 \pm 5.27 \\
+, a, b\end{array}$ & $\begin{array}{c}\text { Acetate } \\
13.19^{\mathrm{a}, \mathrm{b}} \\
(11.79-17.55)\end{array}$ & $\begin{array}{c}\text { Propionate } \\
2.90^{\text {a }} \\
(2.35-3.28)\end{array}$ & $\begin{array}{c}\text { Butyrate } \\
5.77^{\mathrm{a}, \mathrm{b}} \\
(5.38-5.94)\end{array}$ & $\begin{array}{c}\text { BSCFAs } \\
2.33^{+, a, b} \\
(1.97-2.94)\end{array}$ & $\begin{array}{c}\text { Other SCFAs } \\
2.16^{+, a, b} \\
(1.83-2.67)\end{array}$ \\
\hline INU2 & $\underset{*, \mathrm{a}}{47.58^{ \pm}} 14.06$ & $\begin{array}{c}28.28^{\mathrm{a}} \\
(20.05-32.92)\end{array}$ & $\begin{array}{c}4.81^{\mathrm{a}} \\
(3.77-5.93)\end{array}$ & $\begin{array}{c}10.74^{\mathrm{a}} \\
(7.60-18.65)\end{array}$ & $\begin{array}{c}0.37^{*, a} \\
(0.32-0.46)\end{array}$ & $\begin{array}{c}0.36^{*, a} \\
(0.24-0.51)\end{array}$ \\
\hline POWS & $\begin{array}{c}101.10 \pm \\
15.73^{*,+, a, b}\end{array}$ & $\begin{array}{c}40.32 *,+, a, b \\
(39.42-51.55)\end{array}$ & $\begin{array}{c}14.04 *,+, a, b \\
(11.54-19.78)\end{array}$ & $\begin{array}{c}41.66^{*,+, a, b} \\
(34.82-45.80)\end{array}$ & $\begin{array}{c}0.93 *,+, a \\
(0.70-1.29)\end{array}$ & $\begin{array}{c}0.76 *,+, a, b \\
(0.50-1.06)\end{array}$ \\
\hline POOLRP & $\begin{array}{c}105.32 \pm \\
19.75^{*,+, \mathrm{a}, \mathrm{b}}\end{array}$ & $\begin{array}{c}45.56^{*, t, a, b} \\
(40.94-50.31)\end{array}$ & $\begin{array}{c}15.03 *,+, a, b \\
(12.80-23.32)\end{array}$ & $\begin{array}{c}42.17^{*, t, a, b} \\
(33.37-43.71)\end{array}$ & $\begin{array}{c}0.90 *, t, \mathrm{a} \\
(0.65-1.21)\end{array}$ & $\begin{array}{c}0.97^{*,+, a, b} \\
(0.67-1.31)\end{array}$ \\
\hline POLWS & $\begin{array}{c}94.45 \pm 15.41 \\
*,+, a, b\end{array}$ & $\begin{array}{c}34.25 *, a, b \\
(31.13-42.87)\end{array}$ & $\begin{array}{c}13.81 *,+, a, b \\
(12.37-21.63)\end{array}$ & $\begin{array}{c}42.27^{*,+, a, b} \\
(32.58-44.15)\end{array}$ & $\begin{array}{l}1.15^{*,+, a, b} \\
(0.76-1.30)\end{array}$ & $\begin{array}{c}0.73 *,+, a, b \\
(0.45-0.89)\end{array}$ \\
\hline POLTPOMW & $\begin{array}{c}107.44 \pm 8.88 \\
*,+, a, b\end{array}$ & $\begin{array}{c}46.52 *,+, a, b \\
(45.07-50.57)\end{array}$ & $\begin{array}{c}20.43^{*}, t, a, b \\
(16.00-24.83)\end{array}$ & $\begin{array}{c}37.71 *,+, a, b \\
(31.31-39.98)\end{array}$ & $\begin{array}{c}1.96^{+, a, b} \\
(1.60-2.20)\end{array}$ & $\begin{array}{c}1.44^{+} \\
(0.56-2.15)\end{array}$ \\
\hline PEWS & $\underset{*,+, a . b}{98.32 \pm 17.41}$ & $\begin{array}{c}35.51 *,+, \mathrm{a} \\
(31.17-47.60)\end{array}$ & $\begin{array}{c}14.51^{*, t, \mathrm{a}} \\
(10.80-19.70)\end{array}$ & $\begin{array}{c}45.39 *,+, a, b \\
(44.67-46.11)\end{array}$ & $\begin{array}{c}0.61^{*, a} \\
(0.50-0.84)\end{array}$ & $\begin{array}{c}0.39 *, \mathrm{a} \\
(0.25-0.58)\end{array}$ \\
\hline PEWSGM & $\begin{array}{c}103.18 \pm \\
14.30^{*,+, a, b}\end{array}$ & $\begin{array}{c}41.03^{*, t, a} \\
(31.12-50.50)\end{array}$ & $\begin{array}{c}16.46^{*, t, a} \\
(10.58-20.58)\end{array}$ & $\begin{array}{c}46.56^{*, t, a, b} \\
(33.08-52.68)\end{array}$ & $\begin{array}{c}0.84^{*, t, a} \\
(0.48-1.10)\end{array}$ & $\begin{array}{c}0.46^{*, a} \\
(0.37-0.62)\end{array}$ \\
\hline HEBS & $\begin{array}{c}141.08 \pm \\
15.28^{*,+, a, b}\end{array}$ & $\begin{array}{c}51.40^{*,+, a, b} \\
(40.02-53.88)\end{array}$ & $\begin{array}{l}35.26 *,+, a, b \\
(28.34-38.74)\end{array}$ & $\begin{array}{c}50.51^{*, t, a, b} \\
(32.60-62.29)\end{array}$ & $\begin{array}{c}5.41^{+, a, b} \\
(3.99-8.02)\end{array}$ & $\begin{array}{l}4.45^{+, a, b} \\
(2.50-5.80)\end{array}$ \\
\hline HEOLRP & $\begin{array}{c}133.45 \pm \\
15.58^{*,+\mathrm{t}, \mathrm{a}, \mathrm{b}}\end{array}$ & $\begin{array}{c}31.59 * \text { a } \\
(24.99-39.75)\end{array}$ & $\begin{array}{c}20.95 *,+a, b \\
(17.96-30.30)\end{array}$ & $\begin{array}{c}80.90 *,+, a, b \\
(50.12-89.47)\end{array}$ & $\begin{array}{c}1.99^{+, a, b} \\
(0.70-1.46)\end{array}$ & $\begin{array}{c}2.17^{+, a, b} \\
(1.43-2.64)\end{array}$ \\
\hline
\end{tabular}


Table 4. Cont.

\begin{tabular}{|c|c|c|c|c|c|c|}
\hline CC2WS & $\begin{array}{c}102.71 \pm \\
17.32 *,+, a, b\end{array}$ & $\begin{array}{c}38.92 *,+, a, b \\
(31.41-51.13)\end{array}$ & $\begin{array}{c}15.13^{*,+, a, b} \\
(14.28-25.54)\end{array}$ & $\begin{array}{c}47.17^{*,+, a, b} \\
(29.88-49.09)\end{array}$ & $\begin{array}{c}0.85^{*, \mathrm{t}, \mathrm{a}} \\
(0.62-1.26)\end{array}$ & $\begin{array}{c}0.44^{*, a, b} \\
(0.25-0.61)\end{array}$ \\
\hline CC505WS & $\begin{array}{c}110.64 \pm \\
14.39 *,+, \mathrm{a}, \mathrm{b}\end{array}$ & $\begin{array}{c}43.06 *+, \mathrm{ta}, \mathrm{b} \\
(34.91-48.64)\end{array}$ & $\begin{array}{c}17.26^{*,+, a, b} \\
(13.12-28.97)\end{array}$ & $\begin{array}{c}46.11^{*,+, a, b} \\
(42.77-50.71)\end{array}$ & $\begin{array}{l}1.16^{*,+, a, b} \\
(0.79-1.55)\end{array}$ & $\begin{array}{c}0.36^{*, \mathrm{a}} \\
(0.20-0.51)\end{array}$ \\
\hline$p$ overall & $<0.001$ & 0.015 & 0.006 & $<0.001$ & $<0.001$ & $<0.001$ \\
\hline $\begin{array}{l}\text { Values are } \\
\text { (for POLTP } \\
\text { at baseline } \\
\text { after Bonfer } \\
\text { at baseline } \\
\text { after Bonfe } \\
\text { (paired san } \\
\text { (paired sam }\end{array}$ & $\begin{array}{l}\mathrm{V} \text { and HEBS } \\
\text { h), after } 8 \text {-h } \\
\text { s adjustment } \\
\text { h), after } 8 \text {-h } \\
\text { 's adjustmer } \\
t \text {-test or Wi } \\
t \text {-test for par }\end{array}$ & $\begin{array}{l}\text { and SD for pa } \\
4 \text { - for CC2WS } \\
\text { nentation }(\mathrm{t}= \\
\text { multiplicity or } \\
\text { nentation }(\mathrm{t}= \\
\text { or multiplicity } \\
\text { con for non-p } \\
\text { etric or Wilcox }\end{array}$ & $\begin{array}{l}\text { or after } 24-\mathrm{h} \text { f } \\
\text { edman's test); } \\
\text { or after } 24-\mathrm{h} \text { fe } \\
\text { Friedman's tes } \\
\text { metric); b: sigr } \\
\text { signed for non }\end{array}$ & $\begin{array}{l}\text { mentation }(\mathrm{t}= \\
\text { significant diff } \\
\text { mentation }(\mathrm{t}= \\
\text {; a: significan } \\
\text { icant differen } \\
\text { arametric). }\end{array}$ & $\begin{array}{l}\text { (repeated } n \\
\text { (repeated } n \\
\text { erence comb }\end{array}$ & $\begin{array}{l}\text { ametric data } \\
\text { NC }(p<0.05) \\
\text { ares ANOVA } \\
\text { JU2 }(p<0.05) \\
\text { ares ANOVA } \\
\text { d to baseline } \\
\text { ermentation }\end{array}$ \\
\hline
\end{tabular}

Table 5. Molar ratios (\%) of SCFAs at baseline and after $8 \mathrm{~h}$ and $24 \mathrm{~h}$ fermentation.

\begin{tabular}{|c|c|c|c|c|c|}
\hline \multicolumn{6}{|c|}{$\begin{array}{l}\text { Baseline }(t=0 ~ h) \\
\text { Molar Ratio }(\%)\end{array}$} \\
\hline & Acetate & Propionate & Butyrate & BSCFAs & Other SCFAs \\
\hline NC & $\begin{array}{c}46.20 \\
(41.82-54.41)\end{array}$ & $\begin{array}{c}11.70 \\
(11.29-15.87)\end{array}$ & $\begin{array}{c}28.83 \\
(21.56-42.37)\end{array}$ & $\begin{array}{c}2.14^{\dagger} \\
(1.90-5.07)\end{array}$ & $\begin{array}{c}2.81 \\
(2.15-7.68)\end{array}$ \\
\hline INU2 & $\begin{array}{c}50.68 \\
(46.30-53.72)\end{array}$ & $\begin{array}{c}13.05 \\
(10.89-14.81)\end{array}$ & $\begin{array}{c}26.29 \\
(21.11-32.42)\end{array}$ & $\begin{array}{c}3.56^{*} \\
(3.18-5.32)\end{array}$ & $\begin{array}{c}4.59 \\
(2.84-7.95)\end{array}$ \\
\hline POWS & $\begin{array}{c}46.34^{\dagger} \\
(41.52-48.37)\end{array}$ & $\begin{array}{c}8.95^{*, \dagger} \\
(7.28-17.12)\end{array}$ & $\begin{array}{c}38.07^{\dagger} \\
(27.59-45.55)\end{array}$ & $\begin{array}{c}2.17^{\dagger} \\
(1.99-2.35)\end{array}$ & $\begin{array}{c}4.51 \\
(2.60-5.72)\end{array}$ \\
\hline POOLRP & $\begin{array}{c}48.67 \\
(45.64-51.55)\end{array}$ & $\begin{array}{c}7.95^{*,+} \\
(7.41-11.01)\end{array}$ & $\begin{array}{c}35.94^{+} \\
(29.02-42.04)\end{array}$ & $\begin{array}{c}2.48^{\dagger} \\
(2.02-2.69)\end{array}$ & $\begin{array}{c}4.02 \\
(2.68-6.05)\end{array}$ \\
\hline POLWS & $\begin{array}{c}44.91^{\dagger} \\
(40.37-49.66)\end{array}$ & $\begin{array}{c}8.63^{*,+} \\
(8.17-11.75)\end{array}$ & $\begin{array}{c}39.15^{*, \dagger} \\
(34.70-41.18)\end{array}$ & $\begin{array}{c}2.32^{\dagger} \\
(1.92-2.92)\end{array}$ & $\begin{array}{c}4.17 \\
(2.65-5.95)\end{array}$ \\
\hline POLTPOMW & $\begin{array}{c}53.23 \\
(50.29-54.05)\end{array}$ & $\begin{array}{c}9.56 \\
(9.04-13.75)\end{array}$ & $\begin{array}{c}29.25 \\
(26.65-34.85)\end{array}$ & $\begin{array}{c}2.07^{\dagger} \\
(1.84-4.11)\end{array}$ & $\begin{array}{c}3.16 \\
(2.68-5.49)\end{array}$ \\
\hline PEWS & $\begin{array}{c}50.19 \\
(45.62-51.99)\end{array}$ & $\begin{array}{c}9.52 *,+ \\
(8.96-10.86)\end{array}$ & $\begin{array}{c}31.83 \\
(28.39-37.69)\end{array}$ & $\begin{array}{c}2.51^{\dagger} \\
(2.15-2.84)\end{array}$ & $\begin{array}{c}4.64 \\
(2.60-6.56)\end{array}$ \\
\hline PEWSGM & $\begin{array}{c}53.32 * \\
(49.54-55.39)\end{array}$ & $\begin{array}{c}9.51^{*,+} \\
(8.60-10.84)\end{array}$ & $\begin{array}{c}30.90 \\
(28.02-33.52)\end{array}$ & $\begin{array}{c}2.66^{\dagger} \\
(1.95-3.05)\end{array}$ & $\begin{array}{c}4.03 \\
(2.69-5.10)\end{array}$ \\
\hline HEBS & $\begin{array}{c}52.54 \\
(50.72-54.59)\end{array}$ & $\begin{array}{c}10.82 \\
(8.97-13.68)\end{array}$ & $\begin{array}{c}28.17 \\
(26.54-31.40)\end{array}$ & $\begin{array}{c}2.45 \\
(1.94-4.69)\end{array}$ & $\begin{array}{c}4.54 \\
(2.44-6.49)\end{array}$ \\
\hline HEOLRP & $\begin{array}{c}61.00 *,+ \\
(58.26-63.29)\end{array}$ & $\begin{array}{c}8.45^{*,+} \\
(7.41-9.90)\end{array}$ & $\begin{array}{c}24.10^{*} \\
(22.74-27.69)\end{array}$ & $\begin{array}{c}2.15^{\dagger} \\
(1.74-2.64)\end{array}$ & $\begin{array}{c}2.97 \\
(2.22-4.01)\end{array}$ \\
\hline CC2WS & $\begin{array}{c}52.94 * \\
(50.32-55.49)\end{array}$ & $\begin{array}{c}12.04 \\
(9.67-12.69)\end{array}$ & $\begin{array}{c}29.30 \\
(24.37-30.04)\end{array}$ & $\begin{array}{c}2.66 \\
(2.00-3.43)\end{array}$ & $\begin{array}{c}3.66 \\
(2.86-5.67)\end{array}$ \\
\hline CC505WS & $\begin{array}{c}50.72 \\
(46.01-51.65)\end{array}$ & $\begin{array}{c}11.75 \\
(10.59-12.44)\end{array}$ & $\begin{array}{c}31.80 \\
(29.85-32.22)\end{array}$ & $\begin{array}{c}3.14 \\
(2.00-3.78)\end{array}$ & $\begin{array}{c}4.14 \\
(2.98-5.85)\end{array}$ \\
\hline \multicolumn{6}{|c|}{$\begin{array}{c}\text { 8-h Fermentation }(t=8 \mathrm{~h}) \\
\text { Molar Ratio }(\%)\end{array}$} \\
\hline $\mathrm{NC}$ & $\begin{array}{c}\text { Acetate } \\
52.73 \\
(50.42-60.20)\end{array}$ & $\begin{array}{c}\text { Propionate } \\
11.36 \\
(9.36-14.29)\end{array}$ & $\begin{array}{c}\text { Butyrate } \\
22.63^{\text {a }} \\
(20.40-25.86)\end{array}$ & $\begin{array}{c}\text { BSCFAs } \\
6.30^{+} \\
(3.95-8.88)\end{array}$ & $\begin{array}{c}\text { Other SCFAs } \\
4.91^{+} \\
(4.03-5.34)\end{array}$ \\
\hline INU2 & $\begin{array}{c}59.38^{\mathrm{a}} \\
(50.88-64.45)\end{array}$ & $\begin{array}{c}8.96 \\
(8.82-15.10)\end{array}$ & $\begin{array}{c}25.40 \\
(19.33-34.05)\end{array}$ & $\begin{array}{c}0.65^{*, \mathrm{a}} \\
(0.45-1.38)\end{array}$ & $\begin{array}{c}0.62 *, \mathrm{a} \\
(0.37-1.13)\end{array}$ \\
\hline POWS & $\begin{array}{c}46.89^{*,+} \\
(45.82-53.30)\end{array}$ & $\begin{array}{c}14.96^{*, \mathrm{t}, \mathrm{a}} \\
(13.50-19.14)\end{array}$ & $\begin{array}{c}34.93^{*,+} \\
(31.98-39.32)\end{array}$ & $\begin{array}{c}0.93^{*}, \mathrm{a} \\
(0.60-1.37)\end{array}$ & $\begin{array}{c}0.62^{*, \mathrm{a}} \\
(0.36-1.38)\end{array}$ \\
\hline POOLRP & $\begin{array}{c}49.66 \\
(45.82-53.30)\end{array}$ & $\begin{array}{c}15.29^{*, t, \mathrm{a}} \\
(14.60-19.95)\end{array}$ & $\begin{array}{c}31.75 * \\
(28.87-37.07)\end{array}$ & $\begin{array}{c}0.96^{*, \mathrm{a}} \\
(0.87-1.40)\end{array}$ & $0.66^{*, \mathrm{a}}(0.45-1.27)$ \\
\hline POLWS & $\begin{array}{c}47.58^{*, \dagger} \\
(39.72-52.87)\end{array}$ & $\begin{array}{c}16.70^{*,+, \mathrm{a}} \\
(14.73-18.42)\end{array}$ & $\begin{array}{c}32.80 * \\
(29.31-38.77)\end{array}$ & $\begin{array}{c}0.94^{*, \mathrm{a}} \\
(0.79-1.15)\end{array}$ & $\begin{array}{c}0.70 * \mathrm{a} \\
(0.37-1.37)\end{array}$ \\
\hline POLTPOMW & $\begin{array}{c}50.26 \\
(45.94-57.58)\end{array}$ & $\begin{array}{c}19.36 *,+, a \\
(17.02-22.98)\end{array}$ & $\begin{array}{c}29.03 \\
(19.73-31.08)\end{array}$ & $\begin{array}{c}1.32 *,+ \\
(1.09-1.56)\end{array}$ & $\begin{array}{c}0.99 * \text { *a } \\
(0.43-1.64)\end{array}$ \\
\hline PEWS & $\begin{array}{c}47.86^{+} \\
(42.29-53.56)\end{array}$ & $\begin{array}{c}15.41^{\mathrm{a}} \\
(12.32-17.71)\end{array}$ & $\begin{array}{c}33.18^{*} \\
(31.89-39.53)\end{array}$ & $\begin{array}{c}0.77^{*, \mathrm{a}} \\
(0.62-1.02)\end{array}$ & $\begin{array}{c}0.43^{*, \mathrm{a}} \\
(0.30-0.83)\end{array}$ \\
\hline
\end{tabular}


Table 5. Cont.

\begin{tabular}{|c|c|c|c|c|c|}
\hline \multirow{2}{*}{ PEWSGM } & $46.42^{+}$ & $15.90 *,+, a$ & $34.43 *$ & $0.78^{*, \mathrm{a}}$ & $0.53 *$, a \\
\hline & $(44.03-58.02)$ & $(12.83-17.61)$ & (27.07-41.08) & $(0.60-1.24)$ & $(0.33-0.77)$ \\
\hline \multirow{2}{*}{ HEBS } & $34.56^{*,+}$ & $24.18^{*,+, \mathrm{a}}$ & $43.59 *,+$ & $2.24^{+}$ & $1.85^{\mathrm{t}, \mathrm{a}}$ \\
\hline & $(24.70-44.95)$ & $(15.72-28.08)$ & $(25.55-46.70)$ & $(2.16-2.47)$ & $(0.87-2.39)$ \\
\hline \multirow{2}{*}{ HEOLRP } & $36.93 *,+, a$ & $18.14^{*, t, a}$ & $44.56^{*,+, a}$ & $1.15^{*,+, \mathrm{a}}$ & $1.40 *,+\mathrm{a}$ \\
\hline & $(25.38-44.23)$ & (12.87-23.03) & $(34.01-53.36)$ & (0.91-1.78) & $(0.94-2.03)$ \\
\hline CC2WS & $\begin{array}{c}50.00 \\
(43.03-60.96)\end{array}$ & $\begin{array}{c}14.97^{\mathrm{a}} \\
(11.46-17.31)\end{array}$ & $\begin{array}{c}27.95 \\
(26.08-34.19)\end{array}$ & $\begin{array}{c}0.86 * \text {, a } \\
(0.79-1.19)\end{array}$ & $\begin{array}{c}0.34 *, \mathrm{a} \\
(0.31-0.76)\end{array}$ \\
\hline CC505WS & $\begin{array}{c}47.93^{+} \\
(44.30-55.33)\end{array}$ & $\begin{array}{c}14.61^{\mathrm{a}} \\
(12.39-18.38)\end{array}$ & $\begin{array}{c}32.37 * \\
(26.51-34.42)\end{array}$ & $\begin{array}{c}0.89 *, \mathrm{a} \\
(0.69-1.49)\end{array}$ & $\begin{array}{c}0.36^{*, a} \\
(0.25-0.66)\end{array}$ \\
\hline \multicolumn{6}{|c|}{$\begin{array}{c}\text { 24-h Fermentation }(t=24 h) \\
\text { Molar Rratio }(\%)\end{array}$} \\
\hline NC & $\begin{array}{c}\text { Acetate } \\
49.74 \\
(45.86-56.75)\end{array}$ & $\begin{array}{c}\text { Propionate } \\
9.85^{\mathrm{a}} \\
(9.34-10.93)\end{array}$ & $\begin{array}{c}\text { Butyrate } \\
19.85^{\mathrm{a}} \\
(18.89-23.29)\end{array}$ & $\begin{array}{c}\text { BSCFAs } \\
8.38^{+, a} \\
(7.92-9.59)\end{array}$ & $\begin{array}{c}\text { Other SCFAs } \\
7.34^{+, b} \\
(6.41-8.71)\end{array}$ \\
\hline INU2 & $\begin{array}{c}60.56^{\mathrm{a}, \mathrm{b}} \\
(53.61-68.09)\end{array}$ & $\begin{array}{c}8.74 \\
(7.58-15.56)\end{array}$ & $\begin{array}{c}23.16^{\mathrm{b}} \\
(18.02-33.35)\end{array}$ & $\begin{array}{c}0.63 *, \mathrm{a} \\
(0.55-1.51)\end{array}$ & $\begin{array}{c}0.79 *, \mathrm{a} \\
(0.42-1.13)\end{array}$ \\
\hline POWS & $\begin{array}{c}43.42 *,+, b \\
(38.20-47.77)\end{array}$ & $\begin{array}{c}13.54 *, \mathrm{a} \\
(12.36-18.99)\end{array}$ & $\begin{array}{c}40.67^{*,+, b} \\
(34.42-45.00)\end{array}$ & $\begin{array}{c}0.91 *, \mathrm{a} \\
(0.64-1.37)\end{array}$ & $\begin{array}{c}0.83^{*, \mathrm{a}} \\
(0.41-1.27)\end{array}$ \\
\hline POOLRP & $\begin{array}{c}44.23^{+, b} \\
(40.94-50.11)\end{array}$ & $\begin{array}{c}15.76 * \text { *t,a } \\
(13.62-17.83)\end{array}$ & $\begin{array}{c}36.57^{*,+, b} \\
(31.91-40.41)\end{array}$ & $\begin{array}{c}0.90 *, \mathrm{a} \\
(0.61-1.29)\end{array}$ & $\begin{array}{c}0.93 *, \mathrm{a} \\
(0.55-1.55)\end{array}$ \\
\hline POLWS & $\begin{array}{c}40.96^{*,+, b} \\
(34.42-44.62)\end{array}$ & $\begin{array}{c}15.40 *,+, \mathrm{b} \\
(13.70-20.78)\end{array}$ & $\begin{array}{c}42.43^{*,+, b} \\
(34.62-46.90)\end{array}$ & $\begin{array}{c}1.34 * \text { a } \\
(0.70-1.49)\end{array}$ & $\begin{array}{c}0.77 *, \mathrm{a} \\
(0.45-1.10)\end{array}$ \\
\hline POLTPOMW & $\begin{array}{c}44.69^{+, a, b} \\
(41.51-46.43)\end{array}$ & $\begin{array}{c}19.00 *,+, \mathrm{a} \\
(16.01-21.62)\end{array}$ & $\begin{array}{c}34.31^{\mathrm{b}} \\
(30.58-36.42)\end{array}$ & $\begin{array}{c}1.85^{+} \\
(1.39-2.21)\end{array}$ & $\begin{array}{c}1.34^{*, \mathrm{a}} \\
(0.48-2.16)\end{array}$ \\
\hline PEWS & $\begin{array}{c}36.54^{*,+, a, b} \\
(30.43-46.46)\end{array}$ & $\begin{array}{c}14.80^{*, \mathrm{a}} \\
(11.35-17.74)\end{array}$ & $\begin{array}{c}45.21^{*,+, a, b} \\
(37.14-56.21)\end{array}$ & $\begin{array}{c}0.60 *, \mathrm{a}, \mathrm{b} \\
(0.51-0.93)\end{array}$ & $\begin{array}{c}0.34^{*, \mathrm{a}} \\
(0.24-0.65)\end{array}$ \\
\hline PEWSGM & $\begin{array}{c}38.92 *,+, a, b \\
(33.40-46.16)\end{array}$ & $\begin{array}{c}15.56^{*,+, a} \\
(11.71-18.44)\end{array}$ & $\begin{array}{c}45.15^{*,+, a, b} \\
(34.44-52.52)\end{array}$ & $\begin{array}{c}0.76^{*, \mathrm{a}} \\
(0.48-1.00)\end{array}$ & $\begin{array}{c}0.46^{*, a} \\
(0.34-0.60)\end{array}$ \\
\hline HEBS & $\begin{array}{c}35.84^{*,+, \mathrm{a}} \\
(26.89-41.64)\end{array}$ & $\begin{array}{c}24.78^{*, t, a, b} \\
(20.15-27.92)\end{array}$ & $\begin{array}{c}35.10 * \\
(25.41-40.94)\end{array}$ & $\begin{array}{c}3.91^{+} \\
(3.08-5.10)\end{array}$ & $\begin{array}{c}3.17^{+, \mathrm{b}} \\
(1.66-4.41)\end{array}$ \\
\hline HEOLRP & $\begin{array}{c}20.49^{*,+, a, b} \\
(19.59-34.32)\end{array}$ & $\begin{array}{c}14.48^{*,+, \mathrm{a}} \\
(13.28-23.42)\end{array}$ & $\begin{array}{c}60.51^{*, t, a, b} \\
(39.30-63.66)\end{array}$ & $\begin{array}{c}1.52^{*, \dagger} \\
(1.06-2.47)\end{array}$ & $\begin{array}{c}1.74^{*, \mathrm{t}, \mathrm{a}} \\
(0.96-1.91)\end{array}$ \\
\hline CC2WS & $\begin{array}{c}42.52 *,+, a, b \\
(29.66-43.38)\end{array}$ & $\begin{array}{c}16.49^{*,+, a} \\
(13.31-16.65)\end{array}$ & $\begin{array}{c}43.00 *,+, a, b \\
(35.65-47.00)\end{array}$ & $\begin{array}{c}0.92 * \text { a } \\
(0.66-1.52)\end{array}$ & $\begin{array}{c}0.37 * \text { a } \\
(0.22-0.65)\end{array}$ \\
\hline CC505WS & $\begin{array}{c}40.39 *,+, a, b \\
(34.37-42.31)\end{array}$ & $\begin{array}{c}16.18^{*, t, a} \\
(13.18-20.97)\end{array}$ & $\begin{array}{c}43.16^{*, t, a, b} \\
(37.21-47.54)\end{array}$ & $\begin{array}{c}1.14 * \text {,a } \\
(0.65-1.45)\end{array}$ & $\begin{array}{c}0.26^{*,+, a} \\
(0.21-0.50)\end{array}$ \\
\hline$p$ overall & $<0.001$ & 0.272 & 0.004 & $<0.001$ & 0.010 \\
\hline
\end{tabular}

Values are expressed as mean and SD for parametric or median and Q1-Q3 quartiles for nonparametric data (for POLTPOMW and HEBS $\mathrm{n}=4$ - for CC2WS and CC505 $\mathrm{n}=7$ ); *: significant difference compared to NC $(p<0.05)$ at baseline $(\mathrm{t}=0 \mathrm{~h})$, after 8 - $\mathrm{h}$ fermentation $(\mathrm{t}=8 \mathrm{~h})$ or after 24-h fermentation $(\mathrm{t}=24 \mathrm{~h})$ (repeated measures ANOVA after Bonferroni's adjustment for multiplicity or Friedman's test); $t$ : significant difference compared to INU2 $(p<0.05)$ at baseline $(t=0 h)$, after 8 - $h$ fermentation $(t=8 h)$ or after 24-h fermentation $(t=24 h)$ (repeated measures ANOVA after Bonferroni's adjustment for multiplicity or Friedman's test); a: significant difference compared to baseline (Paired Samples $t$-test or Wilcoxon for non-parametric); b: significant difference compared to 8-h fermentation (Paired Samples $t$-test for parametric or Wilcoxon signed for non-parametric).

Baseline $(\mathrm{t}=0 \mathrm{~h})$ average concentration of total VFAs was approximately $3.0 \mu \mathrm{mol} \mathrm{mL} \mathrm{L}^{-1}$ for all treatments and controls, reaching 67.0 and $95.0 \mu \mathrm{mol} \mathrm{mL}^{-1}$ after $8 \mathrm{~h}$ and $24 \mathrm{~h}$ of fermentation, respectively. Fermentation resulted in significantly higher levels of total VFAs in all treatments, compared to baseline (Table 4). Positive control (INU2) induced a significant increase in total VFAs concentration after 8-h and 24-h fermentation compared to NC, whereas all the tested mushrooms resulted in significantly higher total VFAs, compared to both NC and INU2 for the two time points. HEBS and HEOLRP reached the highest total VFAs concentration at the end of the fermentation process (approximately 130-140 $\mu \mathrm{mol} \mathrm{mL}^{-1}$ ) (Table 4 and Figure 2a-d).

The fermentation process resulted in significant concentration increments of the major SCFAs (acetate, propionate, butyrate) in all treatments (Table 4 and Figure 2a-d). Baseline $(t=0 h)$ average concentration of acetate was approximately $1.5 \mu \mathrm{mol} \mathrm{mL}{ }^{-1}$ for all treatments and controls, reaching 
31.0 and $38.0 \mu \mathrm{mol} \mathrm{mL} L^{-1}$ after $8 \mathrm{~h}$ and $24 \mathrm{~h}$ of fermentation, respectively. Acetate concentration in the positive control (INU2) was marginally higher compared to NC at 8 -h $(p=0.050)$ and $24-h$ fermentation $(p=0.053)$ (Table 4), though a higher production rate was observed in INU2 at both time points (Figure 2a-d). All tested mushrooms demonstrated higher concentration (Table 4) and production rate of acetate compared to NC (Figure 2a-d). Furthermore, some of them (POOLRP, POLTPOMW, PEWS, PEWSGM, CC2WS and CC505WS) induced significantly higher levels of acetate compared to the positive control (INU2), detected after only $8 \mathrm{~h}$ of fermentation, whereas others (POWS, POLWS, HEBS) exhibited an analogous effect after $24 \mathrm{~h}$ of fermentation (Table 4).

Baseline $(t=0 \mathrm{~h})$ average concentration of propionate was approximately $0.3 \mu \mathrm{mol} \mathrm{mL}^{-1}$ for all treatments and controls, reaching 11.5 and $16.0 \mu \mathrm{mol} \mathrm{mL}^{-1}$ after $8 \mathrm{~h}$ and $24 \mathrm{~h}$ of fermentation, respectively. Propionate concentration in positive control (INU2) paralleled that of NC at 8 -h $(p=0.265)$ and 24-h fermentation $(p=0.390)$ (Table 4$)$, though a higher change in propionate concentration was observed in INU2 at both time points compared to NC (Figure 2a-d). Nevertheless, all mushrooms induced significantly higher levels of propionate compared to both controls after $8 \mathrm{~h}$ and $24 \mathrm{~h}$ of fermentation, with HEBS exerting more intense changes.

Average concentration of butyrate was approximately $0.9 \mu \mathrm{mol} \mathrm{mL} \mathrm{L}^{-1}$ for all treatments and controls at baseline $(\mathrm{t}=0 \mathrm{~h})$, reaching 23.0 and $39.0 \mu \mathrm{mol} \mathrm{mL} \mathrm{L}^{-1}$ after $8 \mathrm{~h}$ and $24 \mathrm{~h}$ of fermentation, respectively. Though a greater change in baseline butyrate concentration was observed in INU2 compared to NC during the fermentation process (Figure 2a-d), no significant difference of mean butyrate levels was observed between controls at $8 \mathrm{~h}(p=0.161)$ or $24 \mathrm{~h}$ of fermentation $(p=0.460)$ (Table 4). All tested mushrooms demonstrated a significantly higher concentration and production rate of butyrate compared to NC and INU2 after $8 \mathrm{~h}$ and $24 \mathrm{~h}$ of fermentation (Table 4 and Figure 2a-d), except for POLTPOMW compared to INU2 at $8 \mathrm{~h}(p=0.130)$. A remarkable increase in butyrate concentration was observed in the case of HEOLRP $\left(70 \mu \mathrm{mol} \mathrm{mL}^{-1}\right)$ after 24 -h fermentation compared to baseline levels, and $25 \mu \mathrm{mol} \mathrm{mL}{ }^{-1}$ higher than butyrate levels detected in the case of other mushrooms exhibiting notable changes (e.g., HEBS, CC505WS, PEWS).

The fermentation process also resulted in significant higher levels of minor SCFAs, such as branched SCFAs (BSCFAs, sum of iso-butyrate, iso-valerate and iso-caproate) and other SCFAs (e.g., valerate, caproic acid, heptanoic acid) in all treatments compared to baseline (Table 4).

The average concentration of BSCFAs was approximately $0.08 \mu \mathrm{mol} \mathrm{mL} L^{-1}$ at baseline $(t=0 \mathrm{~h})$ for all treatments and controls, reaching 0.9 and $1.4 \mu \mathrm{mol} \mathrm{mL} \mathrm{m}^{-1}$ after $8 \mathrm{~h}$ and $24 \mathrm{~h}$ of fermentation, with the same values being estimated at $0.12,0.6$ and $1.1 \mu \mathrm{mol} \mathrm{mL} L^{-1}$, respectively, in the case of other SCFAs. Branched SCFA production was significantly elevated after $8 \mathrm{~h}$ and $24 \mathrm{~h}$ of fermentation in the case of $\mathrm{NC}$, while a similar pattern was detected in the case of some mushroom/substrate combinations (HEBS, HEOLRP, POLTPOMW). HEBS was the only treatment that induced a significantly greater change of BSCFAs concentration compared to both NC and INU2. On the contrary, the production of BSCFAs was rather limited in the case of the INU2 and P. eryngii mushrooms (PEWS, PEWSGM), with PEWS being the only treatment demonstrating marginally similar levels of BSCFAs after $24 \mathrm{~h}$-fermentation compared to INU2 $(p=0.066)$. The rest of the tested mushrooms exhibited significantly higher concentration of BSCFAs than INU2, but lower than NC after $24 \mathrm{~h}$ of fermentation (Table 4). The production of the other SCFAs was also significantly elevated in the case of NC, with HEBS and HEORLP following a similar pattern; on the other hand, the production of other SCFAs was rather limited in the case of INU2, with P. eryngii (PEWS, PEWSGM) and C. cylindracea (CC2WS, CC505WS) mushrooms showing an analogous effect. P. ostreatus mushrooms had a rather intermediate effect between NC and INU2 (Table 4 and Figure 3a,b).

Molar ratios of SCFAs (i.e., \% total VFA proportion of SCFAs) at baseline and after 8-h and 24-h fermentation are presented in Table 5. In the case of the negative control (NC), significant increments were recorded in molar ratio of BSCFAs and other SCFAs, presumably in expense of molar ratios of butyrate and propionate, but not acetate. Fermentation of prebiotic inulin (INU2) induced the highest molar ratio of acetate among treatments (approximately $60 \%$ of total VFAs produced) and a significant 
reduction in molar ratios of BSCFAs and other SCFAs; in addition, a reduced molar ratio of butyrate was observed at $24 \mathrm{~h}$ compared to 8 -h fermentation.

P. ostreatus mushrooms (POLWS, POOLRP, POLWS, POLTPOMW) resulted in a significant higher molar ratio of propionate after $8 \mathrm{~h}$ of fermentation, with a rather stable molar ratio of acetate and a stable to decreased molar ratio of butyrate (Table 5). After $24 \mathrm{~h}$ of fermentation, a significant drop in molar ratio of acetate and an increase in molar ratio of butyrate were detected, while the molar ratio of propionate remained rather unchanged. Molar ratios of other SCFAs and BSCFAs were reduced during the fermentation of $P$. ostreatus mushrooms, with more limited BSCFAs-related effects in the case of POLTPOMW. Treatments involving P. eryngii mushrooms (PEWS, PEWSGM) also resulted in a significant higher molar ratio of propionate after $8 \mathrm{~h}$ of fermentation, which was maintained until the end of fermentation. Furthermore, a significant drop in molar ratio of acetate with a subsequent significant increase in molar ratio of butyrate (approximately $45 \%$ of total VFAs produced) was observed between $8 \mathrm{~h}$ and $24 \mathrm{~h}$ of fermentation of both mushrooms. Molar ratio of other SCFAs and BSCFAs was significantly reduced during the fermentation of P. eryngii mushrooms, with PEWS inducing a significant decrease in molar ratio of BSCFAs between all-time points of fermentation.

The use of C. cylindracea mushrooms (CC2WS, CC505WS) resulted, also, in a significant higher molar ratio of propionate after $8 \mathrm{~h}$ of fermentation, which was preserved until the end of fermentation. Furthermore, a significant drop in molar ratio of acetate with a subsequent significant increase in molar ratio of butyrate (approximately $43 \%$ of total VFAs produced) was observed between $8 \mathrm{~h}$ and $24 \mathrm{~h}$ fermentation of both mushrooms. The molar ratio of other SCFAs and BSCFAs was significantly reduced during the fermentation of both $C$. cylindracea strains (Table 5).

A more versatile behavior was observed when H. erinaceus mushrooms (HEBS, HEOLRP) were compared (Table 5). In detail, both treatments resulted in a significantly higher molar ratio of propionate after $8 \mathrm{~h}$ of fermentation, which was preserved until the end of fermentation, with HEBS exhibiting the highest final molar ratio of propionate among all treatments (approximately $25 \%$ of total VFAs produced). Mushrooms from both substrates induced a decrease in molar ratio of acetate during fermentation, with a more drastic effect noted in the case of HEOLRP. Furthermore, fermentation of HEOLRP resulted in a significantly increased and scalable response of molar ratio of butyrate, resulting in the highest values among all treatments at the end of the process (approximately $60 \%$ of total VFAs), an effect not observed in the case of HEBS. In fact, molar ratios of major SCFAs (acetate:propionate:butyrate) after $24 \mathrm{~h}$ fermentation were 35:25:35 for HEBS and 20:15:60 for HEOLRP. For minor SCFAs, $24 \mathrm{~h}$ fermentation of HEOLRP resulted in a decreased molar ratio of other SCFAs and -most likely- BSCFAs $(p=0.069)$ compared to the baseline, whereas HEBS induced a significant reduction in molar ratio of other SCFAs at 8-h fermentation with both molar ratios of other SCFAs or BSCFAs almost returning to baseline levels after 24-h fermentation.

Finally, comparison of the same mushroom strain in different substrates (POWS vs. POOLRP, POLWS vs. POLTPOMW, PEWS vs. PEWSGM, HEBS vs. HEOLRP) or of different strains of the same species cultivated in the same substrate (POWS vs. POLWS, CC2WS vs. CC505WS) resulted at a significantly higher production of BSCFAs in POLTPOMW and HEBS compared to POLWS and HEOLRP, respectively. Similarly, a trend for higher production of other SCFAs in HEBS compared to HEOLRP after $24 \mathrm{~h}$ of fermentation was observed, with no significant differences among treatments. The effects of HEBS and POLTPOMW detected on SCFAs profile (e.g., BSCFAs, other SCFAs, propionate) were also verified for the first four runs of the experiment with all substrates available (data not shown).

\section{Discussion}

Mushrooms contain a plethora of bioactive components, including polysaccharides, composed of glucose, mannose, galactose, fucose, arabinose, glucuronic acid and $\beta$-D-glucans, which are considered to have a beneficial effect on human health [33-35]. Although several in vitro gut models have been used to explore the role of dietary fibers on the gut microbiota, to the best of our knowledge, there are only a few studies examining the effects of edible mushrooms (i.e., lyophilized powder from the entire 
fruitbody) on the gut microbiota composition and/or metabolites production [27,29]. For this reason, the aim of this work was to examine the effects of edible mushrooms using an in vitro batch culture fermentation system, inoculated with fecal samples from apparently healthy subjects over 65 years old.

In elderly persons, alterations in the intestinal function are evident, such as increased mucosal membrane permeability, changes in immune function and microbial dysbiosis [26]. Studies have shown decreased intestinal levels of Bacteroides and Bifidobacterium spp. in the elderly populations, whereas an increase in facultative anaerobes, such as streptococci, enterococci and enterobacteria, is evident [36-38]. In recent years, several in vitro and in vivo approaches have attempted to reverse this effect through various dietary formulations, such as probiotics and prebiotics [39-42].

In our study, Pleurotus spp. and C. cylindracea mushrooms induced a significant bifidogenic effect, compared to the baseline. The same effect was further observed for POOLRP and CC505WS after $24 \mathrm{~h}$ of fermentation compared to NC. Recently Zhao, et al. [27] demonstrated that the in vitro fermentation of P. ostreatus and P. eryngii mushrooms promoted the growth of Bifidobacterium spp. This is in line also with several in vitro and animal-based data, which demonstrated a bifidogenic effect of Pleurotus spp. extracts, a phenomenon dependent on the variability among strains and on the chemical composition of growth substrates [43,44].

An increase in bifidobacteria is considered as a marker of intestinal health, and many studies have highlighted their beneficial effect on the prevention of colorectal cancer, colon regularity and acute diarrhea [45]. To the best of our knowledge, there are no published data demonstrating the impact of C. cylindracea mushrooms or extracts on the intestinal Bifidobacterium spp. levels. The potential lactogenic effect of polysaccharide extracts from P. eryngii has been reported in a few studies [44,46,47]. Our results demonstrated that $P$. eryngii mushrooms from both substrates induced a significant increase of lactobacilli compared to NC and baseline after 24-h fermentation, showing an analogous effect to the prebiotic inulin. In contrast, the in vitro fermentation of P. ostreatus and P. eryngii mushrooms did not favor the growth of lactobacilli Zhao, et al. [27]. Likewise, in a different in vitro model the fermentation of P. eryngii mushrooms with fecal inoculum from healthy donors, resulted in no remarkable change in Lactobacillus spp. levels, whereas the ratio of bifidobacteria/lactobacilli/Enterobacteriaceae remained stable during the fermentation process [29]. These contradictory results are probably due to intrinsic factors that influence the bioactive compounds content among strains of the same species, as it is already reported in basidiomycetes [48,49].

F. prausnitzii (Ruminococcaceae) and E. rectale/Roseburia spp. group (Lachnospiraceae) are two of the most dominant butyrate-producers [24]. The levels of the colonic butyrate-producing bacteria are related to host health, since diabetic populations, human colorectal cancer patients, elderly people and IBD patients are characterized by reduced levels of butyrate producers and increased levels of opportunistic pathogens. The E. rectale/Roseburia spp. group is abundant in the gut microbiota, whereas lower numbers appear (with parallel decrease in fecal butyrate concentration) when a high protein and low carbohydrate diet is followed by human volunteers [50]. Notably, in our study, 24-h fermentation of PEWSGM significantly increased the levels of both bacterial groups, whereas POWS, POOLRP, POLWS, PEWS, CC505WS had positive effects in F. prausnitzii population, i.e., a bacterium that has been related to anti-inflammatory properties [51]. On the contrary, these bacterial populations were significantly reduced when no carbohydrate source was provided (negative control).

The Prebiotic Index allows for the comparison of the prebiotic effect of different substrates [52]. It remains a useful tool and it has been applied in the prebiotic investigation of various natural substrates (e.g., almond skins) [53] or processed carbohydrates, such as inulin, fructooligosaccharides, polydextrose and isomaltooligosaccharides [54]. As shown in Figure 1a,b, all mushroom treatments exhibited positive prebiotic indexes after 24 -h fermentation. The highest values were observed in P. eryngii (PEWS) due to its strong lactogenic effect and in C. cylindracea (CC505WS), due to its bifidogenic effect and decrease of Bacteroides spp. levels. The prebiotic inulin and the mushroom PEWS were the only substrates that had significantly increased PIs compared to NC. 
SCFAs are mainly produced from carbohydrate fermentation and have been shown to contribute significantly to host health. The most common SCFAs in the colon (acetate, propionate, butyrate) reduce the luminal $\mathrm{pH}$ resulting in gut microbial alterations and growth inhibition of $\mathrm{pH}$-sensitive pathogens, increase in mineral absorption and influence intestinal motility [18]. Many studies have demonstrated that the fermentation of prebiotic substrates results in increased production of SCFAs [55]. However, variations in the chemical structure of prebiotics are known to affect their utilization by the gut microbiota and, thus, SCFAs production [45]. Acetate, propionate and butyrate are present in the colon in an approximate molar ratio of 60:20:20, respectively, although the amount and relative proportion of each SCFA is depending on the substrate, the microbiota composition and gut transit time [56].

In our study, fermentation process resulted in significant concentration increments of the major SCFAs (acetate, propionate, butyrate) in all substrates. The fermentation of all mushroom-based substrates induced the production of acetate compared to NC for both time points $(8 \mathrm{~h}, 24 \mathrm{~h})$ (Table 4 and Figure 2a-d). Some of the treatments (POOLRP, POLTPOMW, PEWS, PEWSGM, CC2WS and CC505WS) induced significantly higher levels of acetate, compared to the prebiotic control INU2 after $8 \mathrm{~h}$ of fermentation, whereas others (POWS, POLWS, HEBS) exhibited an analogous effect after $24 \mathrm{~h}$ of fermentation. In the present study, a significant drop in molar ratio of acetate with a subsequent significant increase in molar ratio of butyrate was observed for P. eryngii and C. cylindracea mushrooms between $8 \mathrm{~h}$ and $24 \mathrm{~h}$ of fermentation, and the same effect was observed for P. ostreatus mushrooms after $24 \mathrm{~h}$ of fermentation.

In a recent in vitro fermentation study [27], P. ostreatus exhibited higher concentrations of total SCFAs, acetate, propionate and butyrate, compared to the baseline and the control; on the contrary, $P$. eryngii increased the concentrations of all SCFAs compared to the baseline, but they remained significantly lower than the control. Acetate is widely produced by different bacterial groups in the gut, while propionate and butyrate are more substrate-specific [57]. Bifidobacteria are considered as important acetate producers during fermentation of inulin-type fructans, and they participate in cross-feeding interactions with other gut bacterial groups resulting in the production of propionate and butyrate [58].

All mushrooms induced significantly higher levels of propionate, compared to both controls after $8 \mathrm{~h}$ and $24 \mathrm{~h}$ of fermentation, with HEBS exerting more intense changes. In addition, all tested mushrooms also resulted in a significantly higher molar ratio of propionate after $8 \mathrm{~h}$ of fermentation. This remarkable increase of propionate from HEBS fermentation could possibly be explained by the high substrate content in rhamnose and fucose $[59,60]$. In this study, the fermentation of $H$. erinaceus mushrooms (HEBS and HEOLRP) has not yielded a proportional increase in the tested bacterial populations known for their propionate production [50]. Intestinal bacteria may produce propionate via three different pathways, depending on the chemical structure of the available substrate and on their genetic background. The succinate pathway is widely distributed in Bacteroidetes and in some Firmicutes species. The acrylate pathway is more restricted within the gut microbes, whilst the propanediol pathway is activated specifically when deoxy sugars (e.g., fucose and rhamnose) enriched substrates are present. Members of the family Lachnospiraceae, e.g., Blautia spp., as well as Ruminococcus and Roseburia species, were found to follow this pathway [50].

Butyrate can be absorbed by the colonic mucosa, and serve as an energy substrate for intestinal epithelial cells, while it also exhibits anti-cancer, pro-apoptotic and anti-inflammatory properties [22,23]. All tested mushrooms demonstrated a significantly higher concentration and production rate of butyrate, compared to controls after $8 \mathrm{~h}$ and $24 \mathrm{~h}$ of fermentation (Table 4 and Figure $2 \mathrm{a}-\mathrm{d}$ ), whereas a remarkable increase was observed in the case of HEOLRP. In this study several mushroom treatments increased the F. prausnitzii population, whereas PEWSGM significantly increased the levels of both butyrate producing groups. On the contrary, in the case of HEOLRP, we did not notice an outstanding increase of the tested butyrate producers, concomitant to the observed butyrate production. Future studies will include the DNA (e.g., 16S rRNA) sequencing of the gut microbiota examined, in order to obtain more information about other, non-tested, butyrate producers. 
Two main types of colonic microbial fermentation can be distinguished, i.e., the saccharolytic fermentation of carbohydrates, as most microorganisms preferentially use them, and proteolytic fermentation (when carbohydrate sources are depleted) [23]. Branched SCFAs (BSCFAs), e.g., isobutyric and isovaleric acid, are generated by fermentation of branched amino acids, valine, leucine and isoleucine, originating from undigestible protein reaching the colon [61]. In the present study, fermentation resulted in significantly higher levels of BSCFAs and other SCFAs in all treatments compared to baseline. Notably, the production of BSCFAs in the case of NC was significantly elevated after $8 \mathrm{~h}$ and $24 \mathrm{~h}$ of fermentation, due to proteolytic activity, whereas HEBS was the only treatment that induced a significantly greater change of BSCFAs concentration compared to both controls. In addition, PEWS was the only treatment demonstrating marginally similar levels of BSCFAs compared to INU2 after $24 \mathrm{~h}$ fermentation. The production of other SCFAs was also significantly elevated in the case of NC, with HEBS and HEORLP following a similar pattern.

In this study, several rich in $\beta$-glucan mushroom species were studied in terms of their prebiotic potential, most of them with rather unexplored effects in gut microbial dynamics. Furthermore, mushrooms obtained from various cultivation substrates deriving from agricultural and agro-industrial by-products were evaluated, offering new alternatives in their exploitation through their bioconversion into value-added products with functional properties. In our study, lyophilized powder from the entire mushrooms were examined, thus allowing the investigation of possible synergistic effects among different bioactive compounds. Most importantly, the study focused on apparently healthy subjects (fecal donors) over 65 years old, providing a model for exploring the beneficial effects of edible mushrooms in gut microbiota dynamics during aging process. The qPCR methodology, in combination with SCFA quantification, provided a detailed picture of microbial dynamics during fermentation process, while the calculation of Prebiotic Indexes made it possible to account for the physiological variability that characterizes the experimental process of the in vitro fermentation.

Nevertheless, in order to overcome the limitations mainly set by the extent of microbiome analysis, we are planning to further extend and validate our study through the application of other types of fermentation processes (e.g., continuous cultures) and gut microbiota sequencing.

\section{Materials and Methods}

\subsection{Fungal Strains, Cultivation Substrates, Mushroom Production and Glucans Content}

In the present study, six strains of Pleurotus ostreatus (IK 1123 and LGM 22), Pleurotus eryngii (LGAM 216), Hericium erinaceus (LGAM 4514) and Cyclocybe cylindracea (LGAM 951 and LGAM 961) were used. Pure cultures were established from material collected from various habitats in Greece, and are preserved in the fungal Culture Collection of Laboratory of General and Agricultural Microbiology (Agricultural University of Athens, Athens, Greece).

Cultivation substrates and conditions for mushroom production were as previously described $[15,62,63]$. Total and $\alpha$-glucans were measured in quadruplicates by means of a commercial kit (Megazyme Ltd., Wicklow, Ireland); $\beta$-glucans content was calculated by subtraction of $\alpha$-glucans from total glucans. Glucan content was expressed in $\% w / w$ of dry weight (d.w.). The treatments used in this study and their glucan content are presented in Table 1. Mushrooms were lyophilized and pulverized, before the in vitro fermentation process.

\subsection{Faecal Donors' Characteristics}

Fecal donors were apparently healthy subjects ( $>65 \mathrm{yrs})$, meeting the following inclusion criteria: (a) body mass index (BMI) $<30 \mathrm{~kg} \mathrm{~m}^{-2}$, with no recent weight loss and extreme dietary behaviors; (b) no history of gastrointestinal disease, chronic constipation, chronic/acute diarrhea, autoimmune disease, coronary disease, liver and/or kidney malfunction; (c) no consumption of antibiotics two months before the study; and (d) no consumption of probiotics and/or prebiotics and/or dietary fiber supplements two weeks before the study. 
Subjects completed a series of questionnaires in relation to sociodemographic parameters (including age, sex, marital status and education level), smoking habits and medical history. Evacuation characteristics were also recorded for the past 7 days prior to fecal sampling. Body weight and height were measured by a dietician on a levelled platform scale (SECA GmbH, Hamburg, Germany) and a wall-mounted stadiometer (SECA GmbH), to the nearest $0.1 \mathrm{~kg}$ and $0.5 \mathrm{~cm}$, respectively. Body mass index (BMI) was calculated by dividing the weight $(\mathrm{kg})$ by the height $\left(\mathrm{m}^{2}\right)$ [64]. Dietary intake was evaluated through a 3-d food diary, and data were analyzed in terms of energy and nutrient intakes, using the Nutritionist Pro software (version 4.1.0.; Axxya Systems, Stafford, TX, USA). Physical activity assessment was performed by the International Physical Activity Questionnaire Short Form questionnaire validated for the Greek population [65]; duration of sedentary activity (sitting or resting, $\mathrm{h} \mathrm{wk}^{-1}$ ) was also reported [64].

\subsection{Ethical Standards}

The study was conducted according to the guidelines laid down in the Declaration of Helsinki, and under the approval of the Bioethics Committee of Harokopio University, Athens, Greece (62-03/07/2018). Written informed consent was obtained from all fecal donors, prior to their inclusion in the study.

\subsection{Fecal Sample Collection and In Vitro Static Batch Culture Fermentations}

Fecal sample collection was performed as previously described [64]. In detail, participants were given a pre-weighed plastic container to collect and return their whole evacuation the next few days. Stool samples were weighted, homogenized and processed within two hours after defecation.

The in vitro static batch culture fermentation process was performed according to the protocol of Olano-Martin, et al. [66] and Rycroft, et al. [67] with slight modifications. The composition of the basal medium was further modified by reducing or excluding from the recipe some evidence-based ingredients with cytotoxic effect (e.g., hemin, Tween ${ }^{\circledR} 80$, resazurin) $[68,69]$. Based on previous testing, modified basal medium was comparable to standard basal medium in terms of in vitro fermentation potential and microbial dynamics (data not shown).

The modified basal medium consisted of the following ingredients $\left(\mathrm{g} \mathrm{L}^{-1}\right)$ : peptone water (Merck KGaA, Darmstadt, Germany), 2.0; yeast extract (Merck KGaA), 2; $\mathrm{NaCl}, 0.10 ; \mathrm{K}_{2} \mathrm{HPO}_{4}, 0.04$; $\mathrm{KH}_{2} \mathrm{PO}_{4}, 0.04 ; \mathrm{MgSO}_{4} .7 \mathrm{H}_{2} \mathrm{O}, 0.01 ; \mathrm{CaCl}_{2} .2 \mathrm{H}_{2} \mathrm{O}, 0.01 ; \mathrm{NaHCO}_{3}, 2.0 ;$ L-cysteine $\mathrm{HCl}$ (Merck KGaA), 0.50; dehydrated bile (Oxgall ${ }^{\mathrm{TM}}$, BD and Company, Sparks, MD, USA), 0.50; hemin (dissolved in some drops of $\mathrm{NaOH} 1.0 \mathrm{M}$ ) (Fluka, Sigma-Aldrich Chemie N.V., Zwijndrecht, Netherlands), 0.005 [69]; Tween ${ }^{\circledR} 80$ (Pancreac Quimica SA, Barcelona, Spain), 0.2 mL L $^{-1}$ [68] and vitamin K1 (Fluka, Sigma-Aldrich Chemie GmbH, Buchs, Switzerland), $10 \mu \mathrm{L} \mathrm{L}^{-1}$. The medium was $\mathrm{pH}$ controlled at 7.0 with $\mathrm{HCl} 1.0 \mathrm{M}$, volumes of $72 \mathrm{~mL}$ were aliquoted into $100 \mathrm{~mL}$ vessels, sterilized at $121^{\circ} \mathrm{C}$ for $15 \mathrm{~min}$ and transferred into the anaerobic chamber (BACTRON ${ }^{\mathrm{TM}} 1.5$ Anaerobic Environmental Chamber, SHELLAB, Cornelius, OR, USA) for a $12 \mathrm{~h}$ overnight pre-reduction the day before the in vitro static batch culture fermentation process.

At the day of the in vitro experiment, $2 \%(w / v)$ of the lyophilized mushrooms powder was added in the basal medium aliquots. The documented prebiotic inulin was used as positive control for the fermentation procedure (2\% $w / v$, INU2) (Orafti ${ }^{\circledR}$ GR, BENEO-Orafti, Oreye, Belgium), whereas a negative control (NC; basal medium with no carbohydrate source) was also included in the experiment. A fecal slurry $(20 \% w / v)$ was prepared in PBS pH $7.3\left(8.0 \mathrm{~g} \mathrm{~L}^{-1} \mathrm{NaCl}, 0.2 \mathrm{~g}\right.$ $\mathrm{L}^{-1} \mathrm{KCl}, 1.15 \mathrm{~g} \mathrm{~L}^{-1} \mathrm{Na}_{2} \mathrm{HPO}_{4}, 0.2 \mathrm{~g} \mathrm{~L}^{-1} \mathrm{KH}_{2} \mathrm{PO}_{4}$ ) [70] and homogenized in a Stomacher ${ }^{\circledR}$ paddle blender (Seward Laboratory Systems Inc., Bohemia, NY, USA) under normal speed (265 rpm) for approximately $20 \mathrm{~s}$. From this slurry, 10\% (v/v) inocula were transferred into the pre-reduced basal medium aliquots with the tested mushrooms or the controls. The static batch cultures were incubated for $24 \mathrm{~h}$ under anaerobic conditions at $37^{\circ} \mathrm{C}$. Samples were collected at baseline $(0 \mathrm{~h}), 8 \mathrm{~h}$ and $24 \mathrm{~h}$ of fermentation, and stored at $-80{ }^{\circ} \mathrm{C}$ until further analysis (gut microbiota and SCFAs profiling). Due to 
limited quantity of some lyophilized mushrooms, fewer runs were performed for POLTPOMW $(n=4)$, HEBS $(n=4)$, CC2WS $(n=7)$ and CC505WS $(n=7)$.

\subsection{Gut Microbiota Analysis}

Total bacterial load and selected members of gut microbiota were enumerated at baseline $(0 \mathrm{~h})$ and after $24 \mathrm{~h}$ fermentation by real-time quantitative PCR (qPCR) as previously described [64]. Primers and qPCR characteristics of gut microbiota analysis are presented in Table 6.

Table 6. Primers and qPCR characteristics of gut microbiota analysis (adapted by Mitsou, et al. [64]).



Quantitative real-time PCR based on SYBR Green I detection chemistry was used to characterize the gut microbiota using species-, genus- and group-specific primers targeting $16 \mathrm{~S}$ rRNA genes of different bacterial groups and the KAPA SYBR ${ }^{\circledR}$ Fast Master Mix (2×) Universal Kit (Kapa Biosystems Inc., Wilmington, MA, USA) (Table 6) [64]. PCR amplification and detection were performed in a LightCycler ${ }^{\circledR} 2.0$ Real-Time PCR System (Roche Diagnostics GmbH, Mannheim, Germany). PCR reactions (final volume $20 \mu \mathrm{L}$ ) were performed in duplicate, in LightCycler ${ }^{\circledR}$ glass capillaries and contained $10 \mathrm{ng}$ of each fecal DNA preparation $\left(2 \mathrm{ng} \mu \mathrm{L}^{-1}\right), 10 \mu \mathrm{L}$ of KAPA kit, $200 \mathrm{nM}$ of each primer, $0.25 \mu \mathrm{L}$ of Bovine Serum Albumin (BSA $20 \mathrm{mg} \mathrm{mL}^{-1}$, New England Biolabs Inc, Hitchin, UK) for minimization of reagent abstraction on glass capillaries surface and $3.95 \mu \mathrm{L}$ PCR-grade water. The thermal cycling conditions included an initial enzyme activation step at $95^{\circ} \mathrm{C}$ for $3 \mathrm{~min}$, followed by 45 cycles of DNA denaturation at $95^{\circ} \mathrm{C}$ for $3 \mathrm{~s}$, primer annealing at optimal annealing temperature for $20 \mathrm{~s}$ and extension at $72{ }^{\circ} \mathrm{C}$ for the minimum time required for data acquisition at $72{ }^{\circ} \mathrm{C}$, according to instrument guidelines [template size(bp)/25]. For the confirmation of the specificity of the amplification products, the melting curve analysis was performed by slowly cooling the PCRs from $95{ }^{\circ} \mathrm{C}$ to $65^{\circ} \mathrm{C}\left(0.1^{\circ} \mathrm{C} \mathrm{s}^{-1}\right)$ with simultaneous measurement of the SYBR Green I signal intensity. Microbial quantification was based on standard curves of genomic DNA from reference strains with the LightCycler ${ }^{\circledR}$ software version 4.1 (Roche Diagnostics $\mathrm{GmbH}$ ). Data are expressed as $\log _{10}$ copies of $16 \mathrm{~S}$ rRNA gene $\mathrm{mL}^{-1}$ of sample [64]. 


\subsection{Prebiotic Indexes}

Prebiotic Indexes (PIs) were calculated after $24 \mathrm{~h}$ of fermentation of the tested substrates, providing a useful tool for the comparison of prebiotic efficiency [66]. Calculation of PIs was based on quantification of bacteria (copies of $16 \mathrm{~S}$ rRNA gene $\mathrm{mL}^{-1}$ of sample) and the following equation [52]:

$$
\mathrm{PI}=\left(\frac{\text { Bif }}{\text { Total }}\right)-\left(\frac{\mathrm{Bac}}{\text { Total }}\right)+\left(\frac{\mathrm{Lac}}{\text { Total }}\right)-\left(\frac{\mathrm{Clos}}{\text { Total }}\right)
$$

where Bif is Bifidobacterium spp. numbers after $24 \mathrm{~h}$ of fermentation $(t=24)$ /numbers at baseline $(t=0)$, Bac is Bacteroides spp. numbers after $24 \mathrm{~h}$ of fermentation $(t=24) /$ numbers at baseline $(t=0)$, Lac is Lactobacillus group numbers after $24 \mathrm{~h}$ of fermentation $(\mathrm{t}=24)$ /numbers at baseline $(\mathrm{t}=0), \mathrm{Clos}$ is Clostridium perfringens group numbers after $24 \mathrm{~h}$ of fermentation $(t=24)$ /numbers at baseline $(t=0)$ and Total is total bacteria numbers after $24 \mathrm{~h}$ of fermentation $(t=24)$ /numbers at baseline $(t=0)$. Based on the prebiotic index equation, an increase in the population of bifidobacteria and/or lactobacilli is assumed as a positive effect and an increase in bacteroides and/or clostridia is assumed as a negative effect. This prebiotic index equation offers the advantage of normalizing the bacterial population changes in relation to the initial microbial levels, accounting for the physiological variability that characterizes the experimental process of the in vitro fermentation [52].

\subsection{Measurement of SCFAs}

Capillary gas chromatography (GC) was applied for the determination of the short-chain fatty acids (SCFAs) concentrations of the in vitro static batch cultures, according to Mountzouris, et al. [79], as previously described [80]. In detail, samples $(1 \mathrm{~mL})$ were centrifuged at $13.000 \times \mathrm{g}$ for $15 \mathrm{~min}$ at $4{ }^{\circ} \mathrm{C}$, and $300 \mu \mathrm{L}$ of the supernatant were stored at $-80^{\circ} \mathrm{C}$ until analysis. At the day of analysis, supernatants $(300 \mu \mathrm{L})$ were vortexed and centrifuged at $13.000 \times g$ for $5 \mathrm{~min}$ at RT. Subsequently, $85 \mu \mathrm{L}$ of each supernatant were mixed with $10 \mu \mathrm{L}$ 2-ethyl-butyrate $(20 \mathrm{mM}$, internal standard) (2-Ethyl butyric acid 99\%, Sigma-Aldrich Corp., St. Louis, MO, USA) and $5 \mu \mathrm{L}$ hydrochloric acid ( $\mathrm{HCl}, 1 \mathrm{M})$. Samples of $1 \mu \mathrm{L}$ were injected into a gas chromatographer (Agilent 6890 GC System, Agilent Technologies, Santa Clara, CA, USA) with a Supelco Nukol ${ }^{\text {TM }}$ Capillary GC Column (size x I.D. $30 \mathrm{~m} \times 0.25 \mathrm{~mm}$, df $0.25 \mu \mathrm{m}$ ) (Sigma-Aldrich Corp., St. Louis, MO, USA). The concentrations of SCFAs were computed based on instrument calibration with SCFA standard mix (Supelco volatile acid standard mix, Sigma-Aldrich Corp., St. Louis, MO, USA). Total volatile fatty acids (VFAs) and individual SCFAs concentrations were expressed as $\mu \mathrm{mol} \mathrm{mL} \mathrm{m}^{-1}$ of sample and molar ratios (\% of VFAs) of acetate, propionate, butyrate, branched-chain SCFAs (BSCFAs; iso-butyrate, iso-valerate, iso-caproic acid) and other SCFAs (valerate, caproic acid and heptanoic acid) were also calculated. The production rates of total VFAs, major SCFAs (acetate, propionate, butyrate) and minor SCFAs (BSCFAs, other SCFAs) were further calculated by subtracting initial concentration $(t=0 \mathrm{~h})$ of SCFAs from concentrations after $8 \mathrm{~h}$ or $24 \mathrm{~h}$ of fermentation $\left(\Delta \mathrm{C}_{\mathrm{t} 8-0}\right.$ or $\left.\Delta \mathrm{C}_{\mathrm{t} 24-0}\right)$.

\subsection{Statistical Analysis}

The continuous variables are presented as mean values and standard deviation (SD) or median and interquartile range (Q1-Q3). Categorical variables are presented as frequencies (n, \%). Normality of distribution of continuous variables was tested by the Shapiro-Wilk test. Bacterial levels $(\mathrm{t}=0 \mathrm{~h}$ and $\mathrm{t}=24 \mathrm{~h})$ and SCFAs characteristics $(\mathrm{t}=0 \mathrm{~h}, \mathrm{t}=8 \mathrm{~h}$ and $\mathrm{t}=24 \mathrm{~h})$ at each sampling time were compared by one-way ANOVA for parametric data and Kruskal Wallis test with Mann-Whitney test for non-parametric data and prospectively by repeated measures ANOVA (RM-ANOVA) for parametric data and the Friedman test for non-parametric data, after Bonferroni's adjustment for multiplicity. Comparisons of bacterial levels and SCFAs characteristics into each treatment (NC, INU2, POWS, POOLRP, POLWS, POLTPOMW, PEWS, PEWSGM, HEBS, HEOLRP, CC2WS, CC505WS) between the different time periods $(0 \mathrm{~h}, 8 \mathrm{~h}, 24 \mathrm{~h})$ were performed by paired-samples $\mathrm{T}$ test for parametric data 
and Wilcoxon signed ranks test for non-parametric data. For comparisons of PIs and production rates of SCFAs $\left(\Delta \mathrm{C}_{\mathrm{t} 8-0}\right.$ or $\left.\Delta \mathrm{C}_{\mathrm{t} 24-0}\right)$, parametric and non-parametric tests were applied (one-way ANOVA, Kruskal Wallis test, $t$-test, Mann-Whitney test). Correlation analysis between mean PIs and average glucans content of mushrooms were performed by the Spearman's rank correlation test and linear regression analysis was based on $\log _{10}$-transformation of mean PIs values. The software program IBM $^{\circledR}$ SPSS ${ }^{\circledR}$ Statistics version 21 was used for the statistical analysis of the results and the significance threshold was set at 5\% $(p<0.05)$.

\section{Conclusions}

This research highlighted the potential of certain edible mushrooms rich in $\beta$ - glucans as candidate prebiotics. Strains of the genera Pleurotus and Cyclocybe exhibited a beneficial influence on the composition of gut microbiota of apparently healthy subjects over 65 years old (increase of Bifidobacterium spp. and F. prausnitzii populations), whereas all mushrooms elicited increased molar ratio of propionate and butyrate after $24 \mathrm{~h}$ of fermentation. H. erinaceus mushrooms induced the highest changes in SCFAs production. The development of nutritional products for preventing pathological conditions and improving the quality of life is of utmost importance for the consumer's health, for the elderly in particular. The application of in vitro, ex vivo and in vivo methodologies for evaluating the biological activities of selected edible mushrooms rich in $\beta$-glucans is expected to elucidate/establish the health-promoting properties of this type of bioactive compounds, and to pave the way for their use in novel functional food products.

Supplementary Materials: The following are available online, Figure S1a,b: Prebiotic Indexes (PIs) of the tested mushrooms and controls after $24 \mathrm{~h}$ fermentation for the first 4 runs of the in vitro fermentation experiment, Table S1: Prebiotic Indexes (PIs) per subject for each one of the treatments included in this study, Figure S2a-d: Linear regression analysis of $\log _{10}$-transformed mean Prebiotic Indexes (PIs) of the tested mushrooms with average total glucan content $(a, c)$ and average $\beta$-glucan content $(b, d)$ for all available data $(a, b)$ and for the first 4 runs $(c, d)$ of the in vitro fermentation experiment.

Author Contributions: Conceptualization, E.K.M., G.S. and A.K.; Methodology, E.K.M., G.S., E.S., E.K., E.T., G.K., G.B., G.I.Z., K.C.M. and A.K.; Investigation, E.K.M., G.S., E.S., E.K., E.T., G.K. and G.B.; Formal analysis and Data curation, E.K.M.; Writing-Original draft preparation, E.K.M. and G.S.; Writing-Review and editing, G.I.Z., K.C.M., V.P. and A.K.; Supervision: A.K.; Funding acquisition, G.I.Z., V.P. and A.K. All authors have read and agreed to the published version of the manuscript.

Funding: This research was co-funded by the EU and Greek national funds, through the Operational Program Competitiveness, Entrepreneurship and Innovation under the call RESEARCH-CREATE-INNOVATE (T1EDK-03404).

Acknowledgments: We warmly acknowledge the study participants for their contribution.

Conflicts of Interest: The authors declare no conflict of interest. The funders had no role in the design of the study; in the collection, analyses, or interpretation of data; in the writing of the manuscript, or in the decision to publish the results.

\section{References}

1. Krajmalnik-Brown, R.; Ilhan, Z.E.; Kang, D.W.; DiBaise, J.K. Effects of gut microbes on nutrient absorption and energy regulation. Nutr. Clin. Pract. 2012, 27, 201-214. [CrossRef] [PubMed]

2. Tidjani Alou, M.; Lagier, J.-C.; Raoult, D. Diet Influence on the Gut Microbiota and Dysbiosis related to Nutritional Disorders. Hum. Microbiome J. 2016, 1, 3-11. [CrossRef]

3. Wang, M.; Wichienchot, S.; He, X.; Fu, X.; Huang, Q.; Zhang, B. In vitro colonic fermentation of dietary fibers: Fermentation rate, short-chain fatty acid production and changes in microbiota. Trends Food. Sci. Technol. 2019, 88, 1-9. [CrossRef]

4. Visconti, A.; Le Roy, C.; Rosa, F.; Rossi, N.; Martin, T.; Mohney, R.; Li, W.; Rinaldis, E.; Bell, J.; Venter, J.; et al. Interplay between the human gut microbiome and host metabolism. Nat. Commun. 2019, $10,4505$. [CrossRef]

5. Pickard, J.; Zeng, M.; Caruso, R.; Nunez, G. Gut microbiota: Role in pathogen colonization, immune responses, and inflammatory disease. Immunol. Rev. 2017, 279, 70-89. [CrossRef] 
6. Iacob, S.; Iacob, D.; Luminos, L. Intestinal Microbiota as a Host Defense Mechanism to Infectious Threats. Front. Microbiol. 2019, 9, 3328. [CrossRef]

7. Cheng, H.-Y.; Ning, M.-X.; Chen, D.-K.; Ma, W. Interactions Between the Gut Microbiota and the Host Innate Immune Response Against Pathogens. Front. Immunol. 2019, 10, 607. [CrossRef]

8. Belizario, J.; Faintuch, J. Microbiome and Gut Dysbiosis. In Metabolic Interaction in Infection. Experientia Supplementum; Silvestre, R., Torrado, E., Eds.; Springer: Cham, Switzerland, 2018; Volume 109, pp. 459-476.

9. Guirro, M.; Costa, A.; Gual-Grau, A.; Herrero, P.; Torrell, H.; Canela, N.; Arola, L. Effects from diet-induced gut microbiota dysbiosis and obesity can be ameliorated by fecal microbiota transplantation: A multiomics approach. PLoS ONE 2019, 14, e0218143. [CrossRef]

10. Lin, C.-S.; Chang, C.-J.; Lu, C.-C.; Martel, J.; Ojcius, D.; Ko, Y.-F.; Young, J.; Lai, H.-C. Impact of the gut microbiota, prebiotics, and probiotics on human health and disease. Biomed. J. 2014, 37, 259-268.

11. Gibson, G.; Probert, H.; Loo, J.; Rastall, R.; Roberfroid, M.; Gibson, G.R.; Probert, H.M.; Loo, J.V.; Rastall, R.A.; Roberfroid, M.B. Dietary modulation of the human colonic microbiota: Updating the concept of prebiotics. Nutr. Res. Rev. 2005, 17, 259-275. [CrossRef]

12. Pham, V.T.; Seifert, N.; Richard, N.; Raederstorff, D.; Steinert, R.E.; Prudence, K.; Mohajeri, M.H. The effects of fermentation products of prebiotic fibres on gut barrier and immune functions in vitro. Peer J. 2018, 6, e5288. [PubMed]

13. Jayachandran, M.; Xiao, J.; Xu, B. A Critical Review on Health Promoting Benefits of Edible Mushrooms through Gut Microbiota. Int. J. Mol. Sci. 2017, 18, 1934. [CrossRef] [PubMed]

14. Ciecierska, A.; Drywień, M.; Hamulka, J.; Sadkowski, T. Nutraceutical functions of beta-glucans in human nutrition. Rocz. Panstw. Zakl. Hig. 2019, 70, 315-324. [PubMed]

15. Koutrotsios, G.; Larou, E.; Mountzouris, K.C.; Zervakis, G.I. Detoxification of Olive Mill Wastewater and Bioconversion of Olive Crop Residues into High-Value-Added Biomass by the Choice Edible Mushroom Hericium erinaceus. Appl. Biochem. Biotechnol. 2016, 180, 195-209. [CrossRef] [PubMed]

16. Koutrotsios, G.; Kalogeropoulos, N.; Kaliora, A.C.; Zervakis, G.I. Toward an Increased Functionality in Oyster (Pleurotus) Mushrooms Produced on Grape Marc or Olive Mill Wastes Serving as Sources of Bioactive Compounds. J. Agric. Food Chem. 2018, 66, 5971-5983. [CrossRef] [PubMed]

17. Laroche, C.; Michaud, P. New Developments and Prospective Applications for $\beta(1,3)$ Glucans. Recent Pat. Biotechnol. 2007, 1, 59-73. [CrossRef]

18. Besten, G.; van Eunen, K.; Groen, A.; Venema, K.; Reijngoud, D.-J.; Bakker, B. The Role of Short-Chain Fatty Acids in the Interplay between Diet, Gut Microbiota, and Host Energy Metabolism. J. Lipid Res. 2013, 54, 2325-2340. [CrossRef]

19. Takagi, R.; Sasaki, K.; Fukuda, I.; Tanaka, K.; Yoshida, K.-i.; Kondo, A.; Osawa, R. A Single-Batch Fermentation System to Simulate Human Colonic Microbiota for High-Throughput Evaluation of Prebiotics. PLoS ONE 2016, 11, e0160533. [CrossRef]

20. Rowland, I.; Gibson, G.; Heinken, A.; Scott, K.; Swann, J.; Thiele, I.; Tuohy, K. Gut microbiota functions: Metabolism of nutrients and other food components. Eur. J. Nutr. 2017, 57, 1-24. [CrossRef]

21. Hosseini, E.; Grootaert, C.; Verstraete, W.; Van de Wiele, T. Propionate as a health-promoting microbial metabolite in the human gut. Nutr. Rev. 2011, 69, 245-258. [CrossRef]

22. Roberfroid, M.; Gibson, G.; Hoyles, L.; McCartney, A.; Rastall, R.; Rowland, I.; Wolvers, D.; Watzl, B.; Szajewska, H.; Stahl, B.; et al. Prebiotic Effects: Metabolic and Health Benefits. Br. J. Nutr. 2010, 104, S1-S63. [CrossRef] [PubMed]

23. Hamer, H.; De Preter, V.; Windey, K.; Verbeke, K. Functional analysis of colonic bacterial metabolism: Relevant to health? Am. J. Physiol. Gastrointest. Liver Physiol. 2012, 302, G1-G9. [CrossRef] [PubMed]

24. Zhang, T.; Yang, Y.; Liang, Y.; Jiao, X.; Zhao, C. Beneficial Effect of Intestinal Fermentation of Natural Polysaccharides. Nutrients 2018, 10, 1055. [CrossRef] [PubMed]

25. Pham, V.; Mohajeri, H. The application of in vitro human intestinal models on the screening and development of pre- and probiotics. Benef. Microbes 2018, 9, 1-18. [CrossRef]

26. Liu, Y.; Gibson, G.; Walton, G. An In Vitro Approach to Study Effects of Prebiotics and Probiotics on the Faecal Microbiota and Selected Immune Parameters Relevant to the Elderly. PLoS ONE 2016, 11, e0162604. [CrossRef]

27. Zhao, R.; Yang, W.; Pei, F.; Zhao, L.; Hu, Q. In vitro fermentation of six kinds of edible mushrooms and its effects on fecal microbiota composition. LWT-Food Sci. Technol. 2018, 96, 627-635. [CrossRef] 
28. Yu, Z.; Liu, B.; Mukherjee, P.; Newburg, D. Trametes versicolor Extract Modifies Human Fecal Microbiota Composition In vitro. Plant. Food Hum. Nutr. 2013, 68, 107-112. [CrossRef]

29. Vamanu, E.; Gatea, F.; Sârbu, I. In Vitro Ecological Response of the Human Gut Microbiome to Bioactive Extracts from Edible Wild Mushrooms. Molecules 2018, 23, 2128. [CrossRef]

30. Rodrigues, D.; Walton, G.; Sousa, S.; Rocha-Santos, T.; Duarte, A.; Freitas, A.; Gomes, A. In vitro fermentation and prebiotic potential of selected extracts from seaweeds and mushrooms. LWT-Food Sci. Technol 2016, 73, 131-139. [CrossRef]

31. Chaikliang, C.; Wichienchot, S.; Youravong, W.; Graidist, P. Evaluation on prebiotic properties of $\beta$-glucan and oligo- $\beta$-glucan from mushrooms by human fecal microbiota in fecal batch culture. Funct. Food Health Dis. 2015, 5, 395-405. [CrossRef]

32. Jungles, T.; Ruthes, A.; El-Hindawy, M.; Moreno, R.; Zhang, X.; Cordeiro, L.; Hamaker, B.; Iacomini, M. In vitro fermentation of Cookeina speciosa glucans stimulates the growth of the butyrogenic Clostridium cluster XIVa in a targeted way. Carbohydr. Polym. 2017, 183, 219-229. [CrossRef]

33. Wang, Q.; Wang, F.; Xu, Z.; Ding, Z. Bioactive Mushroom Polysaccharides: A Review on Monosaccharide Composition, Biosynthesis and Regulation. Molecules 2017, 22, 955. [CrossRef] [PubMed]

34. Azmi, A.; Shuhaimi, M.; Abd Manap, Y.; Maaruf, A.G. Mushroom as a potential source of prebiotics: A review. Trends Food Sci. Technol. 2009, 20, 567-575.

35. Gargano, M.; Van Griensven, L.; Isikhuemhen, O.; Lindequist, U.; Venturella, G.; Wasser, S.; Zervakis, G.I. Medicinal mushrooms: Valuable biological resources of high exploitation potential. Plant. Biosyst. 2017, 151, 548-565. [CrossRef]

36. Gavini, C. Differences in the Distribution of Bifidobacterial and Enterobacterial Species in Human Faecal Microflora of Three Different (Children, Adults, Elderly) Age Groups. Microb. Ecol. Health Dis. 2001, 13, 40-45. [CrossRef]

37. Mueller, S.; Saunier, K.; Hanisch, C.; Norin, E.; Alm, L.; Midtvedt, T.; Cresci, A.; Silvi, S.; Orpianesi, C.; Verdenelli, M.C.; et al. Differences in fecal microbiota in different European study populations in relation to age, gender, and country: A cross-sectional study. Appl. Environ. Microbiol. 2006, 72, 1027-1033. [CrossRef]

38. Claesson, M.J.; Cusack, S.; O’Sullivan, O.; Greene-Diniz, R.; de Weerd, H.; Flannery, E.; Marchesi, J.R.; Falush, D.; Dinan, T.; Fitzgerald, G.; et al. Composition, variability, and temporal stability of the intestinal microbiota of the elderly. Proc. Natl. Acad. Sci. USA 2011, 108, 4586-4591. [CrossRef]

39. Toward, R.; Montandon, S.; Walton, G.; Gibson, G. Effect of prebiotics on the human gut microbiota of elderly persons. Gut Microbes 2012, 3, 57-60. [CrossRef] [PubMed]

40. Vulevic, J.; Juric, A.; Walton, G.; Claus, S.; Tzortzis, G.; Toward, R.; Gibson, G. Influence of galacto-oligosaccharide mixture (B-GOS) on gut microbiota, immune parameters and metabonomics in elderly persons. Br. J. Nutr. 2015, 114, 1-10. [CrossRef] [PubMed]

41. Guigoz, Y.; Rochat, F.; Perruisseau-Carrier, G.; Rochat, I.; Schiffrin, E.J. Effects of oligosaccharide on the faecal flora and non-specific immune system in elderly people. Nutr. Res. 2002, 22, 13-25. [CrossRef]

42. Bouhnik, y.; Achour, L.; Paineau, D.; Riottot, M.; Attar, A.; Bornet, F.R.J. Four-week short chain fructo-oligosaccharides ingestion leads to increasing fecal bifidobacteria and cholesterol excretion in healthy elderly volunteers. Nutr. J. 2007, 6, 42. [CrossRef] [PubMed]

43. Koutrotsios, G.; Patsou, M.; Mitsou, E.; Bekiaris, G.; Kotsou, M.; Tarantilis, P.; Pletsa, V.; Kyriacou, A.; Zervakis, G.I. Valorization of Olive By-Products as Substrates for the Cultivation of Ganoderma lucidum and Pleurotus ostreatus Mushrooms with Enhanced Functional and Prebiotic Properties. Catalysts 2019, 9, 537. [CrossRef]

44. Synytsya, A.; Míčková, K.; Synytsya, A.; Jablonsky, I.; Spěváček, J.; Erban, V.; Kováříková, E.; Čopíková, J. Glucans from fruit bodies of cultivated mushrooms Pleurotus ostreatus and Pleurotus eryngii: Structure and potential prebiotic activity. Carbohydr. Polym. 2009, 76, 548-556. [CrossRef]

45. Fehlbaum, S.; Prudence, K.; Kieboom, J.; Heerikhuisen, M.; Broek, T.; Schuren, F.; Steinert, R.; Raederstorff, D. In Vitro Fermentation of Selected Prebiotics and Their Effects on the Composition and Activity of the Adult Gut Microbiota. Int. J. Mol. Sci. 2018, 19, 3097. [CrossRef] [PubMed]

46. Ma, G.; Muinde, B.; Zhao, L.; Yang, W.; Pei, F.; Hu, Q. In vivo fermentation of Pleurotus eryngii polysaccharide and its effects on fecal microbiota composition and immune response. Food Funct. 2017, 8, 1810-1821. [CrossRef] [PubMed] 
47. Fu, Z.; Liu, Y. A Potent Pharmacological Mushroom: Pleurotus eryngii. Fungal Genet. Biol. 2016, 6, 1-5. [CrossRef]

48. Lu, J.; Qin, J.-Z.; Chen, P.; Chen, X.; Zhang, Y.-Z.; Zhao, S.-J. Quality Difference Study of Six Varieties of Ganoderma lucidum with Different Origins. Front. Pharmacol. 2012, 3, 57. [CrossRef]

49. Koutrotsios, G.; Kalogeropoulos, N.; Stathopoulos, P.; Kaliora, A.C.; Zervakis, G.I. Bioactive compounds and antioxidant activity exhibit high intraspecific variability in Pleurotus ostreatus mushrooms and correlate well with cultivation performance parameters. World J. Microbiol. Biotechnol. 2017, 33, 98. [CrossRef]

50. Flint, H.; Louis, P.; Duncan, S. The role of the gut microbiota in nutrition and health. Nat. Rev. Gastroenterol. Hepatol. 2012, 9, 577-589. [CrossRef]

51. Sokol, H.; Pigneur, B.; Watterlot, L.; Lakhdari, O.; Bermúdez-Humarán, L.; Gratadoux, J.-J.; Blugeon, S.; Bridonneau, C.; Furet, J.-P.; Corthier, G.; et al. From the Cover: Faecalibacterium prausnitzii is an anti-inflammatory commensal bacterium identified by gut microbiota analysis of Crohn disease patients. Proc. Natl. Acad. Sci. USA 2008, 105, 16731-16736. [CrossRef]

52. Palframan, R.; Gibson, G.R.; Rastall, R.A. Development of a quantitative tool for the comparison of the prebiotic effect of dietary oligosaccharides. Lett. Appl. Microbiol. 2003, 37, 281-284. [CrossRef] [PubMed]

53. Mandalari, G.; Faulks, R.; Bisignano, C.; Waldron, K.; Narbad, A.; Wickham, M. In vitro evaluation of the prebiotic properties of almond skins (Amygdalus communis L.). FEMS Microbiol. Lett. 2010, 304, 116-122. [CrossRef] [PubMed]

54. Ghoddusi, H.; Grandison, M.A.; Grandison, A.; Tuohy, K. In vitro study on gas generation and prebiotic effects of some carbohydrates and their mixtures. Anaerobe 2007, 13, 193-199. [CrossRef] [PubMed]

55. Poeker, S.A.; Geirnaert, A.; Berchtold, L.; Greppi, A.; Krych, L.; Steinert, R.E.; de Wouters, T.; Lacroix, C. Understanding the prebiotic potential of different dietary fibers using an in vitro continuous adult fermentation model (PolyFermS). Sci. Rep. 2018, 8, 4318. [CrossRef]

56. Dalile, B.; Oudenhove, L.; Vervliet, B.; Verbeke, K. The role of short-chain fatty acids in microbiota-gut-brain communication. Nat. Rev. Gastroenterol. Hepatol. 2019, 16, 461-478. [CrossRef]

57. Morrison, D.; Preston, T. Formation of short chain fatty acids by the gut microbiota and their impact on human metabolism. Gut Microbes 2016, 7, 1-12. [CrossRef]

58. De Vuyst, L.; Moens, F.; Selak, M.; Rivière, A.; Leroy, F. Summer Meeting 2013: Growth and physiology of bifidobacteria. J. Appl. Microbiol. 2014, 116, 477-491. [CrossRef]

59. Wu, F.; Zhou, C.; Zhou, D.; Ou, S.; Zhang, X.; Huang, H. Structure characterization of a novel polysaccharide from Hericium erinaceus fruiting bodies and its immunomodulatory activities. Food Funct. 2017, 9, $294-306$. [CrossRef]

60. Sun, P.; Zhang, J.; Tang, C.-h.; Fan, J.-m.; Shi, X.-m.; Pan, Y.-j. Structural investigation of a novel fucoglucogalactan isolated from the fruiting bodies of the fungus Hericium erinaceus. Food Chem. 2007, 104, 451-456.

61. Heimann, E.; Nyman, M.; Pålbrink, A.-K.; Lindkvist-Petersson, K.; Degerman, E. Branched short-chain fatty acids modulate glucose and lipid metabolism in primary adipocytes. Adipocyte 2016, 5, 359-368. [CrossRef]

62. Koutrotsios, G.; Mountzouris, K.C.; Chatzipavlidis, I.; Zervakis, G.I. Bioconversion of lignocellulosic residues by Agrocybe cylindracea and Pleurotus ostreatus mushroom fungi-assessment of their effect on the final product and spent substrate properties. Food Chem. 2014, 161, 127-135. [CrossRef] [PubMed]

63. Zervakis, G.I.; Koutrotsios, G.; Katsaris, P. Composted versus raw olive mill waste as substrates for the production of medicinal mushrooms: An assessment of selected cultivation and quality parameters. BioMed Res. Int. 2013, 2013, 546830. [CrossRef] [PubMed]

64. Mitsou, E.; Kakali, A.; Antonopoulou, S.; Mountzouris, K.; Yannakoulia, M.; Panagiotakos, D.; Kyriacou, A. Adherence to the Mediterranean diet is associated with the gut microbiota pattern and gastrointestinal characteristics in an adult population. Br. J. Nutr. 2017, 117, 1645-1655. [CrossRef] [PubMed]

65. Papathanasiou, G.; Georgoudis, G.; Papandreou, M.; Spyropoulos, P.; Georgakopoulos, D.; Kalfakakou, V.; Evangelou, A. Reliability Measures of the Short International Physical Activity Questionnaire (IPAQ) in Greek Young Adults. Hellenic J.Cardiol. 2009, 50, 283-294.

66. Olano-Martin, E.; Gibson, G.R.; Rastell, R.A. Comparison of the in vitro bifidogenic properties of pectins and pectic-oligosaccharides. J. Appl. Microbiol. 2002, 93, 505-511. [CrossRef]

67. Rycroft, C.; Jones, M.R.; Gibson, G.R.; Rastall, R.A. A comparative in vitro evaluation of the fermentation properties of prebiotic oligosacharides. J. Appl. Microbiol. 2001, 91, 878-887. [CrossRef] [PubMed] 
68. Hamzeloo-Moghadam, M.; Taiebi, N.; Mosaddegh, M.; Tehrani, B.; Esmaeili, S. The effect of some cosolvents and surfactants on viability of cancerous cell lines. RJP 2014, 1, 41-45.

69. Zhang, N.; Huang, X.; Zeng, Y.; Wu, X.; Peng, X. Study on prebiotic effectiveness of neutral garlic fructan in vitro. Food Sci. Hum. Wellness 2013, 2, 119-123. [CrossRef]

70. Palframan, R.; Gibson, G.; Rastall, R. Effect of pH and Dose on the Growth of Gut Bacteria on Prebiotic Carbohydrates in vitro. Anaerobe 2002, 8, 287-292. [CrossRef]

71. Nadkarni, M.; Martin, F.; Jacques, N.; Hunter, N.; Nadkarni, M.A.; Martin, F.E.; Jacques, N.A.; Hunter, N. Determination of bacterial load by real-time PCR using a broad-range (universal) probe and primers set. Microbiology (Reading, Engl.) 2002, 148, 257-266. [CrossRef]

72. Rinttilä, T.; Lyra, A.; Malinen, E.; Krogius, L.; Palva, A. Development of an extensive set of 16S rRNA-targeted primers for quantification of pathogenic and indigenous bacteria in fecal samples by real-time PCR. J. Appl. Microbiol. 2004, 97, 1166-1177. [CrossRef] [PubMed]

73. Ramirez-Farias, C.; Slezak, K.; Fuller, Z.; Duncan, A.; Holtrop, G.; Louis, P. Effect of inulin on the human gut microbiota. Stimulation of Bifidobacterium adolescentis and Fecalibacterium prausnitzii. Br. J. Nutr. 2008, 101, 541-550. [CrossRef] [PubMed]

74. Phong, S.F.; Shanmugavelu, S.; Thayalini, K.; Noraini, S.; Wong, H.K. Detection of Lactobacillus, Bacteroides and Clostridium perfringens in the gastrointestinal contents of chicken fed different diets by real-time PCR. J. Trop. Agric. Sci. 2010, 38, 81-87.

75. Walker, A.; Ince, J.; Duncan, S.; Webster, L.; Holtrop, G.; Ze, X.; Brown, D.; Stares, M.; Scott, P.; Bergerat, A.; et al. Dominant and diet-responsive groups of bacteria within the human colonic microbiota. ISME J. 2011, 5, 220-230. [CrossRef] [PubMed]

76. Feng, J.; Tang, H.; Li, M.; Pang, X.; Wang, L.; Zhang, M.; Zhao, Y.; Zhang, X.; Shen, J. The abundance of fecal Faecalibacterium prausnitzii in relation to obesity and gender in Chinese adults. Arch. Microbiol. 2013, 196, 73-77. [CrossRef]

77. Suau, A.; Rochet, V.; Sghir, A.; Gramet, G.; Brewaeys, S.; Sutren, M.; Rigottier-Gois, L.; Dore, J. Fusobacterium Prausnitzii and Related Species Represent a Dominant Group Within the Human Fecal Flora. Syst. Appl. Microbiol. 2001, 24, 139-145. [CrossRef] [PubMed]

78. Wang, R.F.; Cao, W.W.; Cerniglia, C.E. PCR detection and quantitation of predominant anaerobic bacteria in human and animal fecal samples. Appl. Environ. Microbiol. 1996, 62, 1242-1247. [CrossRef]

79. Mountzouris, K.; Balaskas, C.; Fava, F.; Tuohy, K.; Gibson, G.; Kostas, F. Profiling of composition and metabolic activities of the colonic microflora of growing pigs fed diets supplemented with prebiotic oligosaccharides. Anaerobe 2006, 12, 178-185. [CrossRef]

80. Mitsou, E.; Kougia, E.; Nomikos, T.; Yannakoulia, M.; Mountzouris, K.; Kyriacou, A. Effect of banana consumption on faecal microbiota: A randomised, controlled trial. Anaerobe 2011, 17, 384-387. [CrossRef]

Sample Availability: Samples of the materials used in this study are available from the authors.

(C) 2020 by the authors. Licensee MDPI, Basel, Switzerland. This article is an open access article distributed under the terms and conditions of the Creative Commons Attribution (CC BY) license (http://creativecommons.org/licenses/by/4.0/). 